\title{
Relations of POD modes and Lyapunov exponents to the nonlinear dynamic states in flow over oscillating tandem cylinders
}

Cite as: Phys. Fluids 30, 123602 (2018); https://doi.org/10.1063/1.5060419

Submitted: 20 September 2018 . Accepted: 28 November 2018 . Published Online: 21 December 2018

Meihua Zhang $(\mathbb{D}$, and Zhongquan $\mathrm{C}$. Zheng
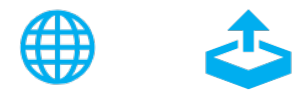

\section{ARTICLES YOU MAY BE INTERESTED IN}

Investigations of data-driven closure for subgrid-scale stress in large-eddy simulation Physics of Fluids 30, 125101 (2018); https://doi.org/10.1063/1.5054835

Machine learning methods for turbulence modeling in subsonic flows around airfoils Physics of Fluids 31, 015105 (2019); https://doi.org/10.1063/1.5061693

Time-domain simulation of ultrasound propagation with fractional Laplacians for lossymedium biological tissues with complicated geometries

The Journal of the Acoustical Society of America 145, 589 (2019); https:// doi.org/10.1121/1.5087826

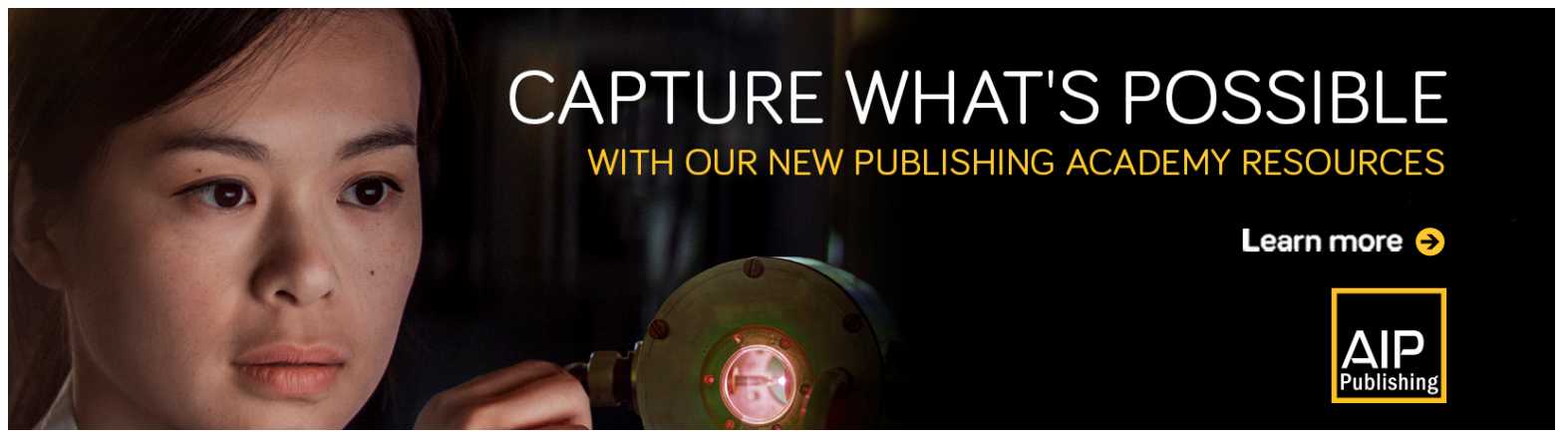




\title{
Relations of POD modes and Lyapunov exponents to the nonlinear dynamic states in flow over oscillating tandem cylinders
}

\author{
Meihua Zhang ${ }^{\text {a) }}$ and Zhongquan C. Zheng \\ Department of Aerospace Engineering, University of Kansas, Lawrence, Kansas 66045, USA
}

(Received 20 September 2018; accepted 28 November 2018; published online 21 December 2018)

\begin{abstract}
Nonlinear dynamic states of flow field are analyzed using the Proper Orthogonal Decomposition (POD) to extract spatial coherent structures, and the temporal development of these spatial structures is further investigated by looking into the largest Lyapunov exponents (LLEs) of the temporal coefficients of the POD modes. Additionally, the LLE of vorticity history at a specific point in the wake is also calculated to compare with the LLEs of the modes in the wake region. The flow field used for the analysis is flow around a two-tandem cylinder system with the downstream cylinder oscillating transversely. The numerical simulation is based on an immersed-boundary method for flow with the Reynolds number 100. Three states of typical nonlinear responses of this system are analyzed: lockin, transitional, and quasiperiodic. The results reveal that the paired POD modes can appear in the energetic modes, although this pattern fades in the transitional state. All the LLEs of the energetic modes in the lock-in state are zero, which means that the energetic modes in the lock-in state are stable. For the transitional state, after a long period of time, the LLEs of the first two energetic modes are close to zero, while the others are positive, showing that only the first two modes are relatively stable and that weak chaotic motions exist in the other modes. The LLEs of all the energetic modes in the quasiperiodic state are no larger than zero after a long period of time, resulting in asymptotical stableness of the energetic modes. Published by AIP Publishing. https://doi.org/10.1063/1.5060419
\end{abstract}

\section{INTRODUCTION}

A rising interest in flow around oscillating tandem cylinders stems from the need to understand the complex spatiotemporal structures and the dynamic behaviors exhibited by this system. It is a complex, multi-variable dynamic system because the center to center distance between the cylinders, the oscillating motion, and the Reynolds number can influence the dynamics and chaos level of this nonlinear flow system. ${ }^{1}$ Therefore, flow around two tandem cylinders can be a good model for understanding the flow physics of other structures because it contains non-linear states that are complicated. Recently, Sumner ${ }^{2}$ provided a review and claimed that the topic of flow around two cylinders would continue to motivate further research because of the complexity of involved fluid dynamics.

This study is focused on flow around two-tandem cylinders with the downstream cylinder oscillating transversely. According to the findings by Yang and Zheng ${ }^{1}$ who discovered how the frequency and spacing influence the behaviors of this multi-variable dynamic system, there exist three states of nonlinear responses: lock-in, transitional, and quasiperiodic. In the present study, three cases representing these three nonlinear states, respectively, are selected for analysis, and these three states are further explored by the Proper Orthogonal Decomposition (POD) ${ }^{3}$ analysis and the largest Lyapunov exponents (LLEs) of the POD mode coefficients. The POD is a convenient tool to represent coherent motions ${ }^{4}$ in the

a)Electronic mail: meihua.zhang@ku.edu flow, and the behaviors of the POD time coefficients have not been fully understood. The development of the LLEs in each state is also analyzed. The scope of this study is to correlate the POD spatial structures and their temporal development, represented by the LLEs of the temporal coefficients, to the non-linear dynamic states and chaos levels of the flow. In addition, the correlation between the LLEs of the POD structures of the flow field and that of a variable (in this case, the vorticity) at a particular point in the flow field are also explored. While a particular flow system is studied in this paper, the procedures presented here can be used to analyze the spatio-temporal development in a wider range of nonlinear flow structures including three-dimensional flow structures.

Flow passing through two identical stationary tandem cylinders is a simper version of this system that is only influenced by the Reynolds number and the center-to-center distance between the two cylinders. Experiments ${ }^{5-7}$ and numerical simulations ${ }^{8-15}$ have long been conducted. It was found that there are three wake-vortex/cylinder interaction regimes: vortex suppression, critical separation, and vortex formation, by changing the center to center distance between the two cylinders in both low Reynolds-number ${ }^{1,8}$ and high Reynolds-number ${ }^{16}$ flows. Xu and Zhou ${ }^{17}$ studied the dominant vortex frequencies in the wake of two stationary tandem cylinders, and the lock-in state was captured. Wang et al. ${ }^{11}$ and Carmo et al. ${ }^{13}$ discussed the stabilities of flow past two stationary cylinders with different center-to-center distances.

There is an ample amount of literature concerning oscillating cylinders in the tandem arrangement. Tanida et al. ${ }^{16}$ 
explored the two-tandem cylinder system by experiments and found that oscillation of the cylinder may become unstable when the cylinder motion and the vortex shedding are synchronized. Based on the finite element method, Li et al. ${ }^{18}$ studied an oscillating cylinder in uniform flow and in the wake of an upstream stationary cylinder. In their study, the lock-in and quasiperiodic response states were found and no clear chaotic behavior was detected in these states. Following the experimental observation by Mahir and Rockwell, ${ }^{19}$ Papaioannou et $a{ }^{20}{ }^{20}$ investigated the flow past two-tandem cylinders oscillating under the same frequency and amplitude with either the same or opposite phases. Peaks at the oscillating frequency $\left(f_{c}\right)$ and its harmonics were found in the lock-in and transitional states, while in the quasiperiodic state, three dominant peaks at $f_{c}, f_{c} / 2$, and $3 f_{c} / 4$ were observed. After that, three distinguishable nonlinear states were found by Yang and Zheng: ${ }^{1}$ lock-in, transitional, and quasiperiodic, with different spacing, frequency, and amplitude conditions. Dominant peaks at $f_{c}$ and its harmonics were shown in the lock-in state. Peaks at the vortex shedding frequency of the system, $f_{n s}$, and the oscillating frequency, $f_{c}$, were both found in the transitional and quasiperiodic states. Bao et al. ${ }^{21}$ studied flow around an oscillating upstream cylinder in tandem with a stationary cylinder by the Boltzmann method. The lock-in and quasiperiodic states were also found in their work.

There have been few efforts exploring the coherent structures, such as the POD modes of vorticity or velocity, which contain dominant physical structures of the system. ${ }^{22,23}$ In addition, the temporal development of the coherent structures, which shows the dynamic development of the system, has not been fully investigated. Important questions regarding a nonlinear flow system are yet to be answered, including what the energetic structures are and how these structures are developing in time (stably or unstably), what levels of chaotic motion these coherent structures contain, and how the nonlinear states observed relate to the spatio-temporal behaviors of the flow structures. This study is intended to use the tandem-cylinder system as an example to answer these questions. In this study, it is justifiable to use Takens'24 time-delay theory to calculate the LLE of each of the time coefficients corresponding to each of the POD modes. This is because Takens' theory can be applied to the time series data to investigate its temporal development, assuming that the time-delayed coordinates construct a state space which is metrically equivalent to contain the original attractor. The method by Rosenstein ${ }^{25}$ is implemented to calculate LLEs that can extend the embedding dimension to below Takens' requirement of greater than two times of the flow dimension.

In the present study, the simulation of flow around a two-tandem-cylinder system is carried out by an improved immersed-boundary method implemented with the porousmedium type model. ${ }^{26}$ The spatial coherent structures are extracted by the POD method. This method was proposed independently by several researchers ${ }^{27,28}$ and introduced in fluid dynamics by Holmes. ${ }^{29}$ It has been used to analyze flows of grooved channels and circular cylinders, ${ }^{30}$ turbulent flows, ${ }^{31,32}$ and reacting flows. ${ }^{33}$ The POD analysis expands the unsteady flow solution into a series summation of each spatial-only POD mode with a temporal coefficient. Thus, the spatial coherent flow structures are represented by the POD modes, while the time evolutions of these structures are represented by their corresponding temporal coefficients. To understand the dynamic behavior of each coherent structure, the largest Lyapunov exponent (LLE) is calculated for the time coefficient of each mode. A system containing at least one positive Lyapunov exponent is identified to be unstable or chaotic. ${ }^{25,34,35}$ Based on the Lyapunov-exponent analysis, Huang et al. $^{36}$ studied the unsteady flow separation control. Guha and Udwadia ${ }^{37}$ analyzed the nonlinear dynamics of wave interactions in multilayered flows. The current study is to use numerical simulation results to perform the POD and LLE analyses, for investigating the temporal behaviors of the POD coherent structures and correlating them with the nonlinear states of the two-tandem-cylinder system.

It has been brought to our attention that a relatively new POD method, named the spectral POD method, has been proposed by Towne et $a l .{ }^{38}$ However, the regular POD method in this study is still popular and widely used nowadays. The method presented in the current paper can analyze the dynamic behavior of the energy-ordered decomposed modes by the regular POD method, which is useful for detecting the dynamic development of energy-containing structures in flow.

In the following content, the numerical scheme and method of the two-tandem-cylinder model are described in Sec. II. A brief overview of POD and calculation of LLEs are explained next in Sec. III. Then the results of POD and LLE analysis for the three cases, each corresponding to one of the three nonlinear states, are presented and discussed in Sec. IV. Finally, conclusions are made in Sec. V.

\section{PROBLEM DESCRIPTION AND NUMERICAL SIMULATION}

An immersed-boundary method is effective in simulating flow around a moving structure because no body-conforming mesh is required around objects in motion. In this study, an improved direct-forcing immersed-boundary method ${ }^{39}$ is used where the solid cylinders are considered as porous media 26,40 with a large resistivity. The model equations are the NavierStokes equations for incompressible flow, with the modified Zwikker-Kosten (Z-K) equation ${ }^{41}$ for flow inside the cylinder body. All the equations and variables are nondimensionalized with the incoming speed and the diameter of the cylinder. The modified governing equations for incompressible, unsteady, viscous fluid flow are written as

$$
\begin{gathered}
\frac{\partial \boldsymbol{u}}{\partial t}+\boldsymbol{u} \cdot \nabla \boldsymbol{u}=-\nabla p+\frac{1}{R e} \nabla^{2} \boldsymbol{u}+\boldsymbol{f}, \\
\nabla \cdot \boldsymbol{u}=0,
\end{gathered}
$$

where $\boldsymbol{f}$ is the body-force term representing the virtual boundary force and $R e$ is the Reynolds number defined as $\rho U_{\infty} D / \mu$, where $U_{\infty}$ is the free stream velocity and $D$ is the diameter of the cylinder. In order to restrict the flow to be two-dimensional, the simulation is performed at the Reynolds number of 100, which is the same Reynolds number as in the work of Yang and Zheng. ${ }^{1}$ The flow, both outside and inside the cylinders, 
can be simulated by the same format of the above governing equations, with the definition of the forcing term as

$$
\boldsymbol{f}= \begin{cases}-\sigma(\boldsymbol{u}-\boldsymbol{V}), & \text { inside the solid body, } \\ 0, & \text { outside the solid body }\end{cases}
$$

where $\sigma$ is the dimensionless flow resistivity of the cylinders in the flow and $V$ is the moving velocity of the objects. For the upstream cylinder, $V$ is zero; for the downstream cylinder, $V$ is the oscillating velocity of the cylinder. The equations for fluid flow and porous media are solved simultaneously, without a need to specify the interface boundary conditions.

The simulation domain and the configuration of the problem are shown in Fig. 1, where a pair of equal-sized cylinders in tandem arrangement are placed in a uniform, incompressible, viscous flow. In this study, the upstream cylinder is stationary, and the downstream cylinder is oscillating periodically in the crossflow direction. The computational domain size for the two-dimensional study is $[0,38.4] \times[0,25.6]$, where the length and width of the domain are nondimensionalized by the cylinder diameter, $D$.

The center of the upstream cylinder is located $8 D$ from the inlet flow boundary to reduce the inlet effect and $30.4 D$ away from the outlet boundary to ensure unstrained vortex wake development. The location of the downstream cylinder is determined by $S$, which is the center-to-center distance between the two cylinders. In this study, $S=2$ and 4 are specified, which are corresponding to the vortex suppression and critical distance, respectively. ${ }^{1}$ The distance between the centerline of the cylinders and the upper and lower boundaries of the computational domain is 12.8 . The size of the uniform, nonmoving grid used in this study is $\Delta x=\Delta y=0.025$ for all the computational cases. The check for grid-independent solution has been carried out in our previous work. ${ }^{39}$ The same boundary conditions are used as in the previous work. ${ }^{1}$ In this study, the computational scheme is a low-storage third-order RungeKutta scheme for time, ${ }^{42}$ the fifth-order WENO-Z method for the convection terms, ${ }^{43}$ and the second-order central difference for the viscous terms. The incompressibility condition is satisfied by solving a Poisson equation for pressure correction using MUDPACK. ${ }^{39,44}$

For the upstream stationary cylinder, the velocity on the surface is zero. For the transversely oscillating downstream

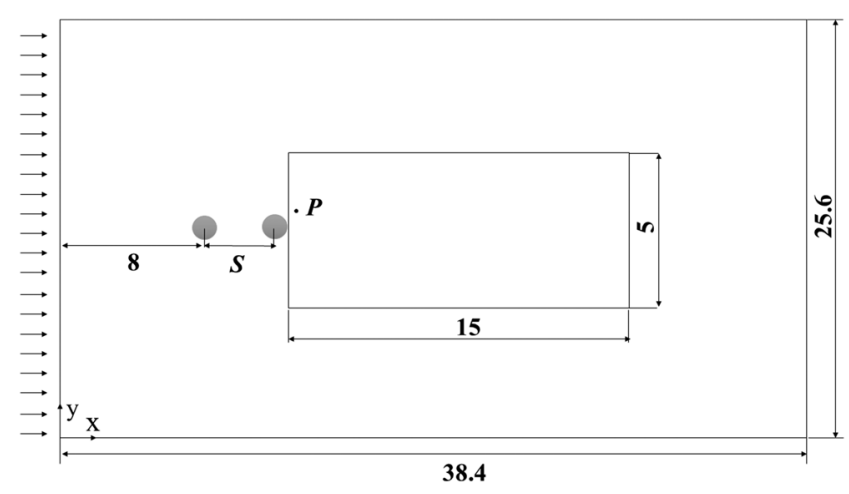

FIG. 1. Sketch of the simulation domain and selected domain for POD. cylinder, the sinusoidal heaving motion is specified as

$$
y(t)=h \sin \left(2 \pi f_{c} t\right),
$$

where $h$ is the heaving magnitude, $f_{c}$ is the oscillating frequency, and $t$ is the time.

The computational scheme has been verified and validated with numerous computational and experimental data. ${ }^{26,40,45}$

\section{MATHEMATICAL FORMULATION OF POD AND LLEs}

\section{A. Mathematical formulation of POD}

A property of the flow field can be constituted as

$$
W(x, t)=\bar{W}(x)+w(x, t),
$$

where $W$ is a variable of flow, which can represent velocity, pressure, vorticity, density, and so on. In this study, vorticity and velocity are used to analyze the flow dynamics. In Eq. (5), $\bar{W}$ is the mean part and $w$ is the time-varying part. As the mean flow represents steady flow, this study focuses on the fluctuating part. The POD modes in this paper are only performed on the fluctuating field, $w$.

After the numerical simulation is conducted, the flow variable, $w$, has a discretized solution in space and time. The starting point of the POD method is a temporal sequence of data field arranged in matrix $X$,

$$
X=\left[w_{1} w_{2} \ldots w_{N}\right]
$$

where $N$ is the number of time snapshots of the recorded time history of the unsteady flow simulation data. Each flow field, $w_{k}$, contains the property of the flow at the $k$ th time instant, with the time interval fixed between each time instant. In the POD method, the flow field can be decomposed into spatially orthogonal modes with corresponding time coefficients $^{46}$

$$
w(x, t)=\sum_{k=1}^{N} \alpha_{k}(t) \varphi_{k}(x),
$$

where $\varphi_{k}(x)$ are the POD modes, representing the coherent structures of the flow, and $\alpha_{k}(t)$ are the time coefficients of the modes. With the orthonormality of $\varphi_{k}(x)$, the time coefficients can be obtained as

$$
\alpha_{k}(t)=\int_{\mathrm{X}} w(x, t) \varphi_{k}(x) d x .
$$

By investigating the behavior of time coefficients, $\alpha_{k}(t)$, i.e., their LLEs (to be discussed later), the stability of each mode can be analyzed.

We need to find a sequence of orthonormal $\varphi_{k}(x)$ in a way that the approximation of using the POD series to represent the flow field is as good as possible, or optimized, in a least-squares sense. To solve this optimization problem, a temporal correlation matrix is calculated first with the method of snapshots 46

$$
C=\int w\left(x, t_{i}\right) \cdot w\left(x, t_{j}\right) d x,
$$

where $i, j=1,2, \ldots, N$. The eigenvalue problem, $C Y=\lambda Y$, is then solved. The POD mode associated with the greatest 
eigenvalue is the optimal vector to characterize the ensemble of snapshots. The $i$ th POD mode can then be constructed as $\varphi_{i}=X Y_{i}$.

In the POD analysis, the energy contained in the data is defined as the sum of the eigenvalues, and the energy percentage captured by the $i$ th POD mode is given by $\lambda_{i} / \sum_{k=1}^{N} \lambda_{k}$, which is also referred to as the energy of each mode. The cumulative energy of the first $i$ POD modes can be represented by $\sum_{k=1}^{i} \lambda_{k} / \sum_{k=1}^{N} \lambda_{k}{ }^{22}$

The wake region behind the oscillating cylinder is most interesting to show the coherent vortical structures and their related flow physics. Therefore, a selected domain of the near wake behind the oscillating cylinder is used for analysis, in the current study, as $[8.5+\mathrm{S}, 23.5+\mathrm{S}] \times[10.3,15.3]$ shown in Fig. 1. The larger domain outside this area is only needed to ensure simulation accuracy, as indicated in the domain-size influence check in our previous work. ${ }^{1}$

\section{B. The largest Lyapunov exponent for $\alpha_{k}(t)$}

Lyapunov exponents are the average exponential rates of convergence or divergence of nearby orbits in the phase space, which are used as a key to indicate the chaos level of a dynamic system. ${ }^{47,48}$ Particularly, the largest Lyapunov exponent (LLE) characterizes the system, and the existence of a positive Lyapunov exponent is an evidence of chaos. In this study, the time coefficients, $\alpha_{k}(t)$, of coherent structures (the POD modes) are a series of values sampled at time intervals. The chaos levels of the coherent structures can thus be analyzed by calculating the LLEs of $\alpha_{k}(t)$. The behaviors of LLEs in this particular problem are expected to relate to the three identified non-linear states of the two-tandem-cylinder system: lock-in, transitional, and quasiperiodic. ${ }^{1}$ A particular interest is to look into the stability or chaos levels of the POD energetic modes by computing the LLEs of the POD mode coefficients.

Nonlinear time-series analysis can extract meaningful information of a dynamic system. This framework relies on the concept of reconstruction of the state space of a system; that is, the one-dimensional time series needs to be reconstructed to a finite-dimensional state space to reflect the dynamic characteristics of the system. ${ }^{49}$ Reconstruction of state space can be processed by lagging the data ${ }^{50}$ and turning $\alpha_{k}(t)$ into a series of vectors. For clarity, in the following discussion in this section, $\alpha(t)$ represents any of $\alpha_{k}(t)$ from the POD series, and $\alpha^{i}(t)$ represents a component of the reconstructed vector from $\alpha(t)$.

To reconstruct the time series, the lag time, $\tau$, and the embedding dimension, $m$, need to be calculated first. ${ }^{32}$ If the $\operatorname{lag} \tau$ was too small, $\alpha^{i}$ and $\alpha^{i+\tau}$ would be indistinguishable. An appropriate selection of $\tau$ is to make $\alpha^{i}$ and $\alpha^{i+\tau}$ sufficiently independent. Here, the mutual information method ${ }^{51}$ is used to calculate the lag, instead of the method based on the Fourier transform. ${ }^{34}$ This method is widely used in the recent literature. For example, Kodba et al. ${ }^{52}$ calculated the lag of a time series produced by a driven resonant circuit, Tamil and Samuel ${ }^{53}$ determined the lag of a temperature time series, and Perc ${ }^{54}$ analyzed the dynamics of human gait.

To introduce the mutual information method, assume two discrete systems, $\left\{r^{1}, r^{2}, \ldots, r^{i}, \ldots\right\}$ and $\left\{q^{1}, q^{2}, \ldots, q^{i}, \ldots\right\}$, represented by $R$ and $Q$, respectively. The marginal entropies resulted from these two systems are

$$
\begin{aligned}
H(R) & =-\sum P_{R}\left(r^{i}\right) \log _{2} P_{R}\left(r^{i}\right), \\
H(Q) & =-\sum P_{Q}\left(q^{j}\right) \log _{2} P_{Q}\left(q^{j}\right), \\
H(R, Q) & =-\sum \sum P_{R, Q}\left(r^{i}, q^{j}\right) \log _{2} P_{R, Q}\left(r^{i}, q^{j}\right),
\end{aligned}
$$

where $P_{R}\left(r^{i}\right)$ is the probability of $r^{i}$ in $R, P_{Q}\left(q^{j}\right)$ is the probability of $q^{j}$ in $Q$, and $P_{R, Q}$ is the joint probability of $\left(r^{i}, q^{j}\right)$ in $R$ and $Q$. The mutual information is defined as

$$
I(R, Q)=H(R)+H(Q)-H(R, Q),
$$

where the probabilities for each of the dataset are obtained by the histogram method ${ }^{55}$ based on Sturges' formula ${ }^{56}$ for constructing a number of bins.

For the assigned datasets, $r^{i}, q^{j}=\left[\alpha^{i}, \alpha^{i+\tau}\right]$, the mutual information, $I(\tau)=I(R, Q)$ of $\alpha^{i}$ and $\alpha^{i+\tau}$, is a measure of the mutual dependence between them. By calculating $I(\tau)$ with $\tau$ from 1 to $n(<N)$, the first minimum of $I\left(\alpha^{i}, \alpha^{i+\tau}\right)$ occurs at the optimal $\tau$. For the length of the time series of $\alpha(t)$ about 20000 , usually a value of $n$ at a few hundreds is sufficient to achieve the first minimum, a fact to be presented in Sec. IV. After $\tau$ is obtained, the embedding dimension $m$ can subsequently be calculated with the Cao method. ${ }^{57}$ In this method, first define

$$
a(i, l)=\frac{\left\|A_{i}(l+1)-A_{i}^{\text {nearest }}(l+1)\right\|}{\left\|A_{i}(l)-A_{i}^{\text {nearest }}(l)\right\|},
$$

where $A_{i}(l+1)$ is the $i$ th reconstructed vector with embedding dimension $l+1, A_{i}^{\text {nearest }}(l+1)$ is the nearest neighbor of $A_{i}(l+1)$ with embedding dimension $l+1$, and $\|\cdot\|$ is a measure of Euclidian distance. Then define

$$
E(l)=\frac{1}{N-l \tau} \sum_{i=1}^{N-l \tau} a(i, l)
$$

Obviously, $E(l)$ only depends on the embedding dimension and the lag. To investigate its variation from dimensions $l$ to $l+1$, the following is defined:

$$
E 1(l)=\frac{E(l+1)}{E(l)},
$$

where $E 1(l)$ will stop changing when $l$ is larger than some value, say, $l_{0}$; then, $l_{0}+1$ is the embedding dimension, $m$.

Based on the lag and embedding dimension, at time $i$, the reconstructed time series $Z_{i}$ is expressed as

$$
Z_{i}=\left(\alpha^{i} \alpha^{i+\tau} \ldots \alpha^{i+(m-1) \tau}\right)
$$

The reconstructed trajectory $Z$, which is an $M \times m$ matrix, can be expressed as

$$
Z=\left(\begin{array}{llll}
Z_{1} & Z_{2} & \ldots & Z_{M}
\end{array}\right)^{T}
$$

where $M=N-(m-1) \tau$, with $N$ as the number of time snapshots in POD and also as the length of the time series $\alpha(t)$.

Once the matrix $Z$ has been constructed for $\alpha(t)$, its LLE is determined based on the mean growth rate of the 
separation of the nearest neighbors using the procedure described in the literature. ${ }^{34}$ The procedure includes, after reconstructing attractor dynamics using the method of delays, finding nearest neighbors, constraining temporal separation, measuring average separation of neighbors, and finally using least squares to fit a logarithmic line to obtain the LLE value. The details and reasoning of each step are presented in Ref. 34. Following this procedure, different time series, $\alpha(t)$, can result in different embedding dimensions and lag times. Therefore, for each different POD mode, the time coefficient may not necessarily contain the same embedding dimension and lag time.

It should be noted that the above process to determine the LLE is performed for each of the POD coefficient $\alpha_{k}(t)$ and the LLE of vorticity history at a specific point. By calculating the LLEs of the time coefficients, the chaos level of each of the corresponding energetic modes can be obtained, and by calculating the LLE of the vorticity history at point $P$ in the flow field, the chaos level at a specific point can be obtained. This also enables us to investigate whether there is a correlation between the stability/chaos level at a specific point in the flow field and those of the POD modes of the flow field in the nearby region.

\section{POD STRUCTURES, LYAPUNOV EXPONENTS, AND THE NONLINEAR DYNAMICS STATES}

In our previous work, ${ }^{1}$ three nonlinear states were found for the tandem-cylinder system: lock-in, transitional, and quasiperiodic, which were also identified by Papaioannou et al. ${ }^{10}$ In the following discussions, the nonlinear dynamic behaviors are further analyzed using POD and LLEs for each of the POD modes. The coherent structures from POD for vorticity and the corresponding temporal coefficients for each of the POD modes are selected to analyze the nonlinear dynamic mechanism.

Time histories, power spectra, and phase portraits are plotted for temporal coefficients of vorticity and velocity POD modes, in order to investigate dynamic states of the POD modes. Then, the Lyapunov exponent analysis is performed to explain the level of chaos of each of the POD vorticity coherent structures. The chaos levels of each POD mode for the vorticity field and that of vorticity at a specific point are compared. In this study, we focus on the dynamics of the most energetic modes because the contribution of higher modes with less energy to the whole system may be ignored. Velocity histories are also recorded at a point that is $0.8 D$ downstream of the center and $0.8 D$ above the center line in the transverse direction of the downstream cylinder (point $P$ shown in Fig. 1), which typically represents the combined wake effects from the two cylinders. The velocity phase portrait and the vorticity history at point $P$ will be shown for each state. Note that the velocity phase portrait and the vorticity history at point $P$ for each state include the mean components of the corresponding variables.

The lock-in, transitional, and quasiperiodic states depend on the distance between the cylinders, $S$, the oscillating amplitude of the downstream cylinder, $h$, and the oscillating frequency of the downstream cylinder $f_{c}$ normalized by the natural frequency $f_{n s}$ of the corresponding stationary tandem cylinder system. Three typical nonlinear states identified by Yang and Zheng ${ }^{1}$ are selected for the study here. The conditions for the lock-in state are $S=2, h=0.15$, and $f_{c}=1.3 f_{n s}$; for the transitional state are $S=4, h=0.35$, and $f_{c}=0.9 f_{n s}$; and for the quasiperiodic state $S=2, h=0.15$, and $f_{c}=1.7 f_{n s}$. For each case, when the simulation reaches 30 oscillating cycles, five oscillation cycles (from $31 T$ to $35 T$, where $T=1 / f_{c}$ ) are chosen for LLEs analysis. To detect the development of the LLEs, when the simulation reaches 100 oscillation cycles, five oscillation cycles (from $81 T$ to $85 T$ ) and twenty oscillation cycles (from $81 T$ to $100 T$ ) are extracted. The first early $5 T$ and second late $5 T$ periods of data are used to explain the development of the LLEs. The last $20 T$ periods of data are used for POD analysis, such as energy distribution and vorticity coherent structures and Lyapunov exponent calculation. To ensure the data-number convergence of the POD modes, two different time intervals, 0.025 and 0.05 , in the time snapshot data are tested. The $\mathrm{L}_{2}$-norm differences ${ }^{22}$ for the first ten modes are calculated in each of the datasets. The results show that the maximum $\mathrm{L}_{2}$-norm difference is below $0.3 \%$ for the time interval equal to 0.025 in each case. Thus, the time interval in data between two successive snapshots of 0.025 is used.

\section{A. The lock-in state}

When the system is in the vortex suppression regime with spacing $S=2$, a small oscillating amplitude $h=0.15$, and an oscillating frequency $f_{c}=1.3 f_{n s}$, the lock-in state is obtained. Figure 2(a) shows the velocity portrait of the downstream wake at point $P$, where the velocity portrait is a clean,

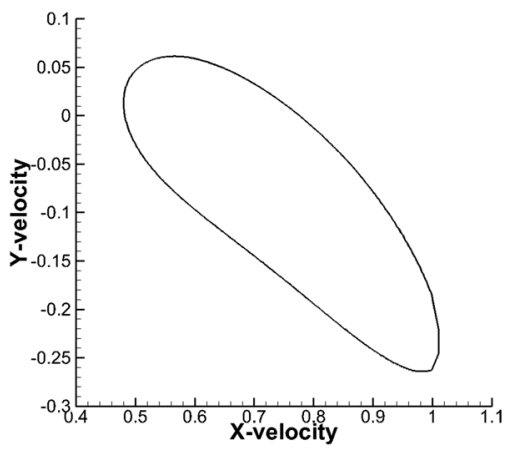

(a)

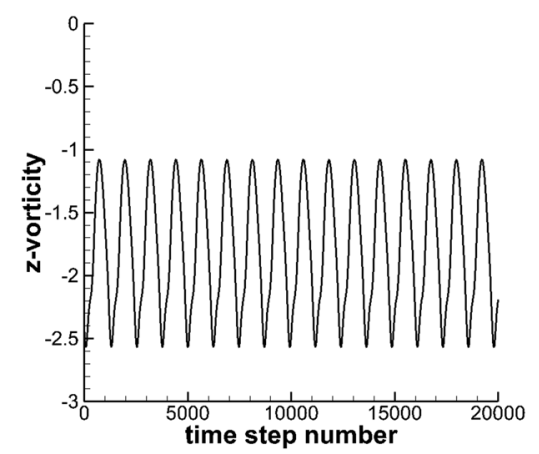

(b)
FIG. 2. Lock-in state: (a) Velocity phase portrait at point $P$ and (b) vorticity time history at point $P$. 
TABLE I. Energy levels of the POD modes and their respective contributions to the total energy in the lock-in state.

\begin{tabular}{lccccrrrr}
\hline \hline Modes & 1 & 2 & 3 & \multicolumn{1}{c}{5} & \multicolumn{1}{c}{5} & \multicolumn{1}{c}{6} & \multicolumn{1}{c}{ ( } \\
\hline Energy of each mode (\%) & 39.93 & 39.42 & 6.43 & 6.28 & 3.41 & 3.38 & 0.43 & 0.43 \\
Cumulative energy (\%) & 39.93 & 79.35 & 85.78 & 92.06 & 95.47 & 98.85 & 99.28 & 99.71 \\
\hline \hline
\end{tabular}

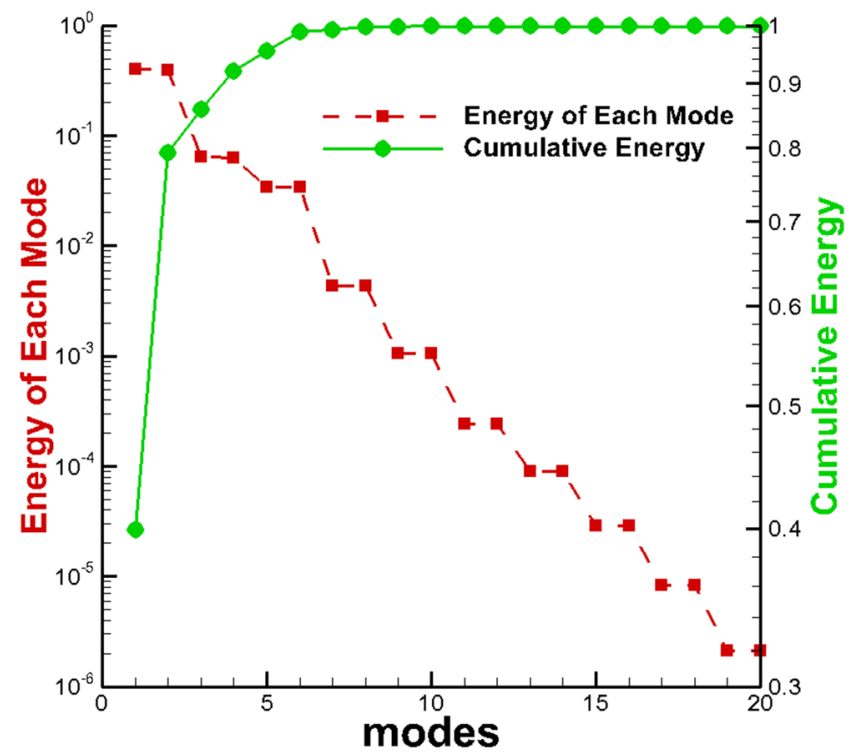

FIG. 3. Energy distributions of the first 20 POD modes in the lock-in state.

closed orbit. The time history of vorticity at point $P$ is shown in Fig. 2(b) where a regular periodicity can be observed. The first eight modes, corresponding to the first eight largest eigenvalues which represent the most energetic modes, are listed in Table I with their percentages of energy contribution and cumulative energy. The energy of each mode and cumulative energy versus the number of the POD modes are shown in Fig. 3, where only the first 20 modes are shown. Clearly, the first two modes dominate and comprise almost $80 \%$ of the total energy of the motion. The first eight modes collectively contain more than $99.5 \%$ of the energy. Thus, within these modes, we have almost captured the entire spatial structure of the flow field. Also shown in Fig. 3, the odd and even modes are found to be paired with close energy levels for the first several modes, similar to those found in the literature. ${ }^{58}$

The first eight flow structures of the POD modes and their mode coefficients are plotted in Fig. 4. The paired modes (modes 1 and 2) have very similar structures, and one is just a shift from the other in the streamwise direction. It also shows that the first pair of modes reveal a top-bottom mirrored symmetry with respect to the centerline of the statistic cylinder (and the domain). The top-bottom symmetric structures appear as a traveling-wave-like flow structure in the streamwise direction. 28,59 The temporal coefficients of these two modes, in Fig. 4(b), show a regular periodicity. After the Fourier transform of the temporal coefficients, we find that the dominant

(a)

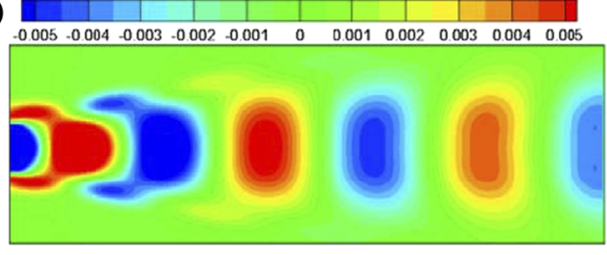

mode1

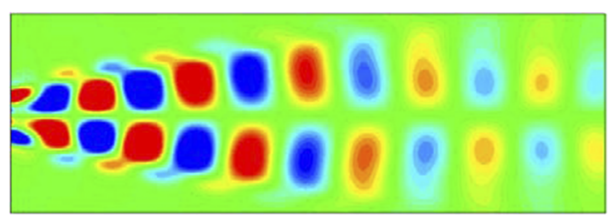

mode3

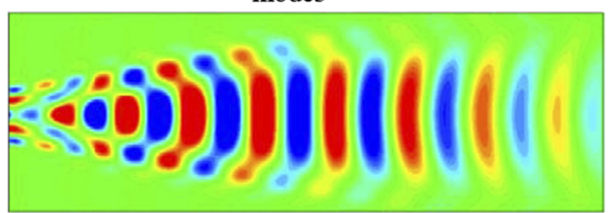

mode5

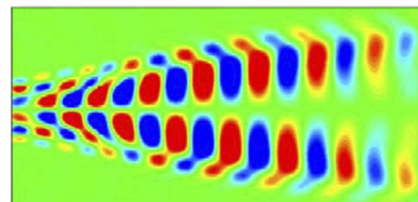

mode 7

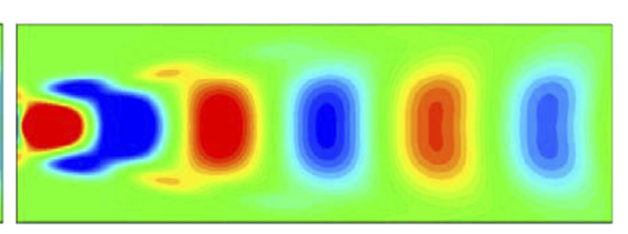

mode2

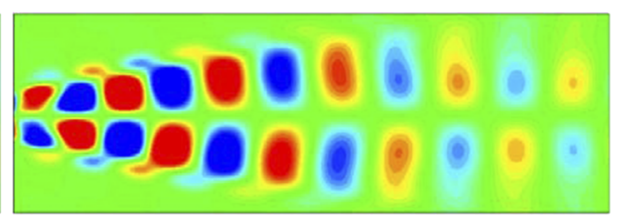

mode4

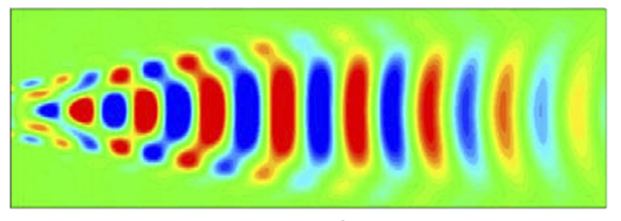

mode6

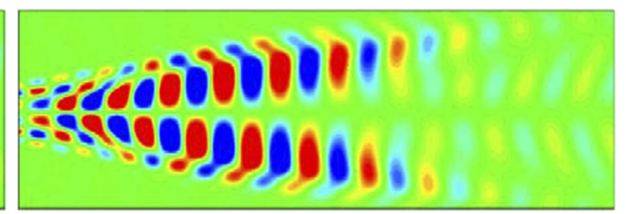

mode8
FIG. 4. Lock-in state: (a) spatial structures of vorticity POD modes (modes 1 to 8 from top to bottom), (b) temporal coefficient time histories of vorticity POD modes, (c) power spectra of the temporal coefficients of vorticity POD modes. 
(b)
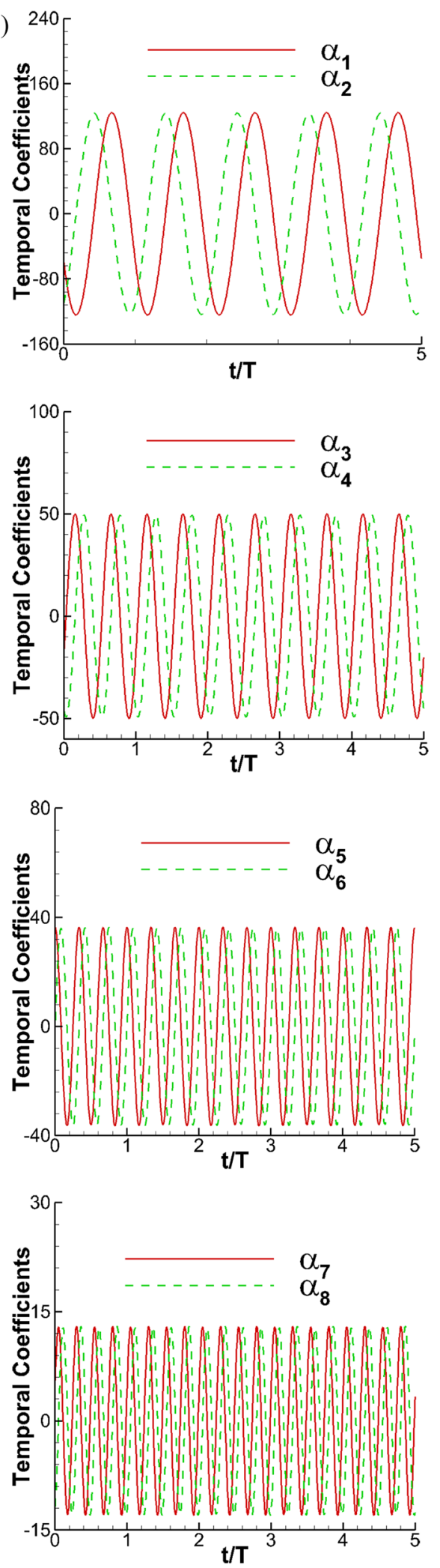

(c)
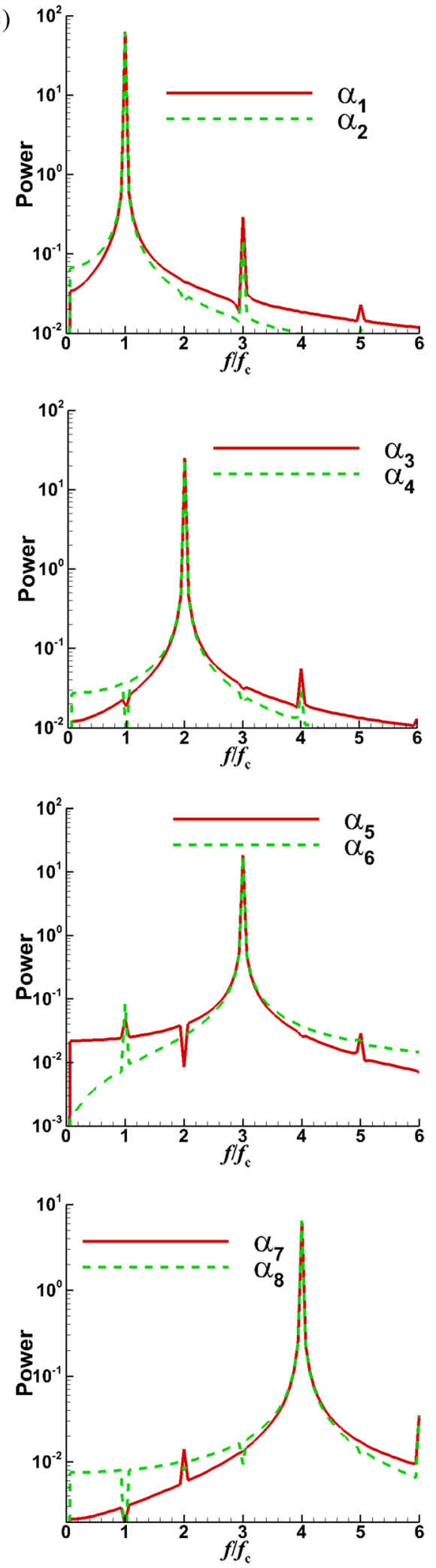

FIG. 4. (Continued).

frequency is the same as the cylinder oscillating frequency $f_{c}$. Thus, the first two dominant modes contain a fundamental frequency corresponding to the oscillating frequency. The second pair of modes (modes 3 and 4) exhibits a top-bottom antisymmetry, with bifurcation and dissipation seen clearly in these two modes. The temporal coefficients also show a regular periodicity, with a dominant frequency found to be
$2 f_{c}$. The third pair (modes 5 and 6 ) again shows a top-bottom symmetry, with structures that are spatial harmonics of those shown in the first pair. In the same way, the fourth pair of modes (modes 7 and 8) shows antisymmetry as the second pair. The symmetric and antisymmetric properties of the wake result from nonlinear interactions of the wake and the symmetric forcing. In addition, the temporal coefficients of paired 


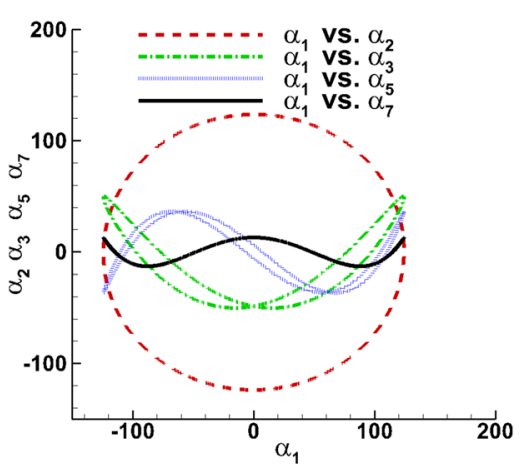

(a)

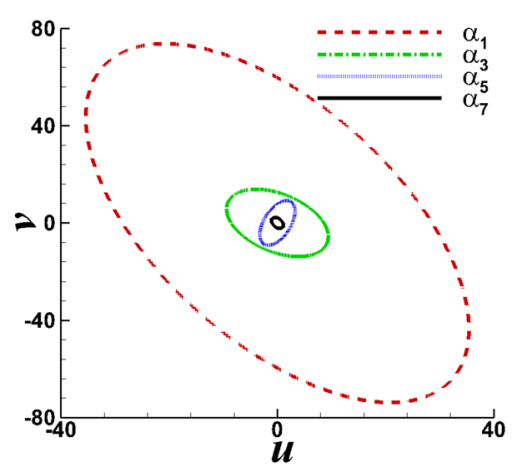

(b)
FIG. 5. Phase portraits of (a) temporal coefficients of vorticity POD modes and (b) temporal coefficients of velocity POD modes, in the lock-in state.

TABLE II. LLE for each vorticity mode in the lock-in state.

\begin{tabular}{lcccccccc}
\hline \hline Temporal coefficients & $\alpha_{1}$ & $\alpha_{2}$ & $\alpha_{3}$ & $\alpha_{4}$ & $\alpha_{5}$ & $\alpha_{6}$ & $\alpha_{7}$ & $\alpha_{8}$ \\
\hline Lag $\tau$ (time units) & 1.5 & 1.5 & 0.75 & 0.75 & 0.45 & 0.45 & 0.25 & 0.25 \\
Embedding dimension $m$ & 3 & 3 & 3 & 3 & 3 & 3 & 3 & 3 \\
LLE (31T-35T) & 0 & 0 & 0 & 0 & 0 & 0 & 0 & 0 \\
LLE (81T-85T) & 0 & 0 & 0 & 0 & 0 & 0 & 0 & 0 \\
LLE (81T-100T) & 0 & 0 & 0 & 0 & 0 & 0 & 0 & 0 \\
\hline \hline
\end{tabular}

modes 1 and 2, i.e., $\alpha_{1}$ and $\alpha_{2}$, have a period $T$, while pairs of $\alpha_{3}$ and $\alpha_{4}, \alpha_{5}$ and $\alpha_{6}$, and $\alpha_{7}$ and $\alpha_{8}$ have periods $T / 2, T / 3$, and $T / 4$, respectively. In the frequency domain, each pair of modes contains different frequencies, with the main frequencies at $f_{c}, 2 f_{c}, 3 f_{c}$, and $4 f_{c}$, respectively, for pairs $1,2,3$, and 4 .

In the lock-in state, the vorticity spectra have clear peaks at $f_{c}$ and its integer multiples as found by Yang and Zheng ${ }^{1}$ and Papaioannou et al. ${ }^{10}$ What was not discovered in the previous studies is that by doing the POD analysis, the spatial modes (coherent structures) along with the frequency-domain behaviors of their corresponding temporal coefficients can be seen clearly.
The phase portraits of the vorticity POD temporal coefficients of higher modes versus that of the first mode are plotted in Fig. 5(a). The first pair is apparently a circle. The higher modes show trajectories of closed cycles pertaining to the super harmonic modes in the lock-in state.

We also performed POD and plotted the coefficients for streamwise $(u)$ and vertical $(v)$ velocities, which showed the same properties of the vorticity POD temporal coefficients as those in Fig. 4(a). So here we omit those plots. Instead, the phase portraits of these time coefficients are shown in Fig. 5(b) and the closed orbits are found. Because the temporal coefficients of velocity POD are also paired in the same way as those of vorticity POD modes in the lock-in state, only $\alpha_{1}, \alpha_{3}, \alpha_{5}$,

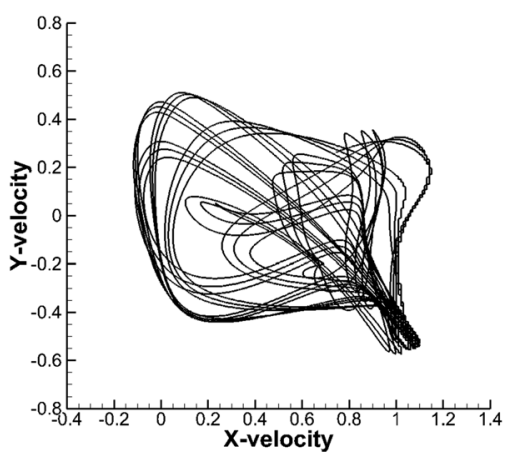

(a)

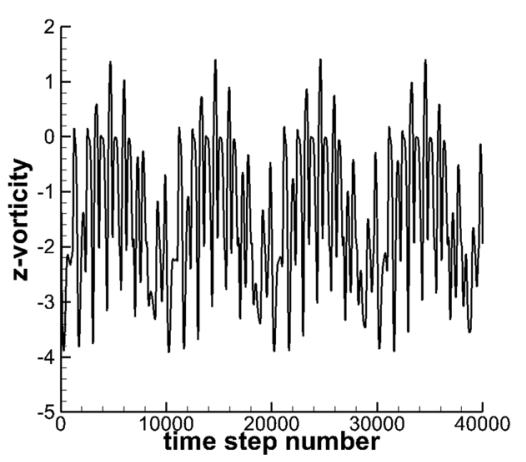

(b)
FIG. 6. Transitional state: (a) Velocity phase portrait at point $P$ and (b) vorticity time history at point $P$.

TABLE III. Energy levels of the POD modes and their respective contributions to the total energy in the transitional state.

\begin{tabular}{lcccccccrrr}
\hline \hline Modes & 1 & 2 & 3 & 4 & 5 & 6 & 7 & 8 & 9 & 10 \\
\hline Energy of each mode (\%) & 35.22 & 33.97 & 4.79 & 3.55 & 3.4 & 2.7 & 1.76 & 1.46 & 1.31 & 1.27 \\
Cumulative energy (\%) & 35.22 & 69.19 & 73.98 & 77.53 & 80.93 & 83.63 & 85.93 & 86.85 & 88.16 & 89.43 \\
\hline \hline
\end{tabular}




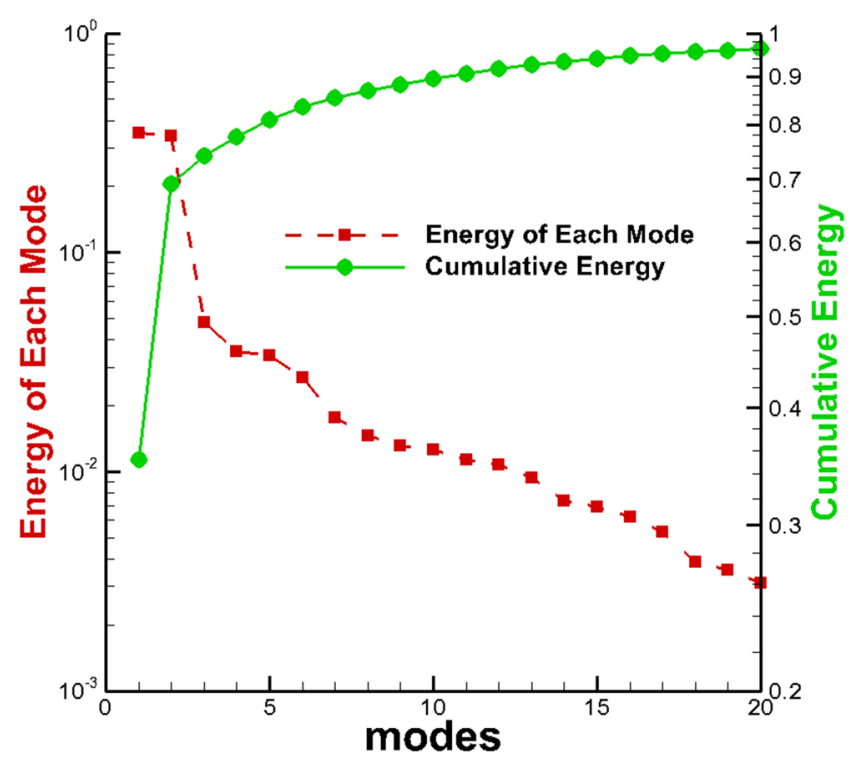

FIG. 7. Energy distributions of the first 20 POD modes in the transitional state.

and $\alpha_{7}$ of $u$ and $v$ are plotted. It can be seen that the trajectories of the temporal coefficients for each of the modes are all in the shape of cleanly closed ellipses.
To investigate the chaos level in the POD coherent structures, we calculate LLEs for the vorticity POD temporal coefficients by the method presented in Sec. III. The transient development of the LLEs is evaluated by using the early first $5 T$, the late $5 T$, and the last $20 T$ time coefficient data. $^{60}$ The results are shown in Table II. The LLE of the vorticity history at point $P$ is also calculated to compare with the LLEs of each field mode. In this case, at point $P$, $\tau=1.5, m=3$, and LLE $=0$. LLEs for all the modes at the early $5 T$, late $5 T$, and last $20 T$ are zero in the lockin state. It demonstrates that, when the simulation becomes period, the LLEs remains to be zero all the time in the lock-in state. This means that there is no chaos existing in the lock-in state and all the energetic modes in the lock-in state are stable. The embedding dimensions of all the modes are also the same as that of point $P$. The lag of point $P$ is the same as that of the first two energetic modes. That is, the lag time is mostly controlled by the highest energy modes.

It is also noted that the lag time of the paired modes is the same. The lag time of modes 1 and 2 is 1.5 , that of modes 3 and 4 is 0.75 , that of modes 5 and 6 is 0.45 , and that of modes 7 and 8 is 0.25 , with a trend of decreasing towards the higher modes. (a)
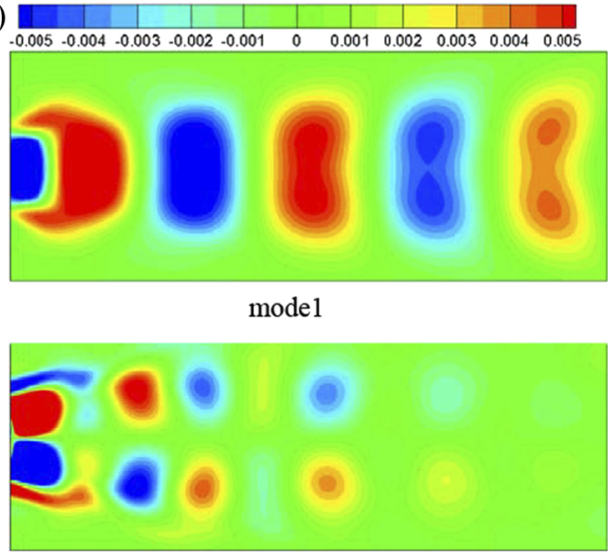

mode3

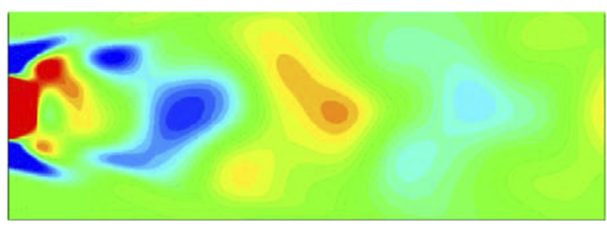

mode5

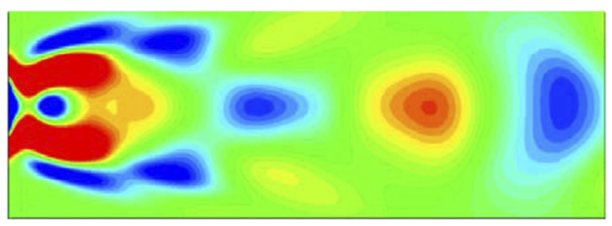

mode7

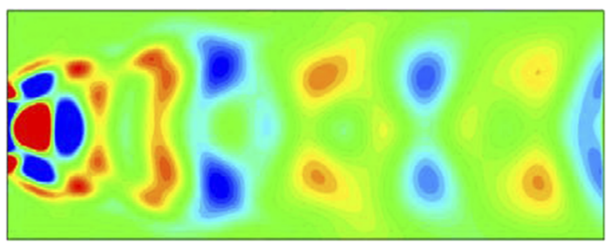

mode9

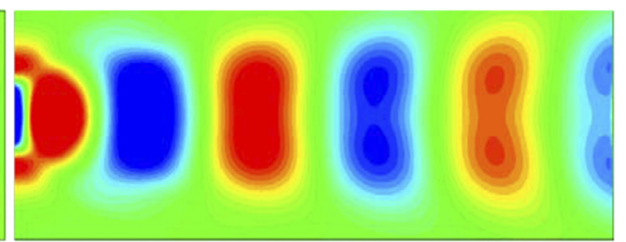

mode2

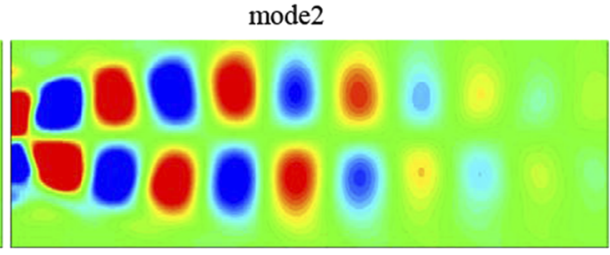

mode4

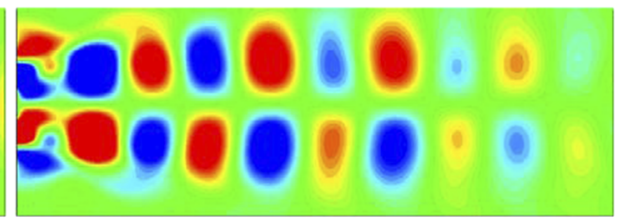

mode6

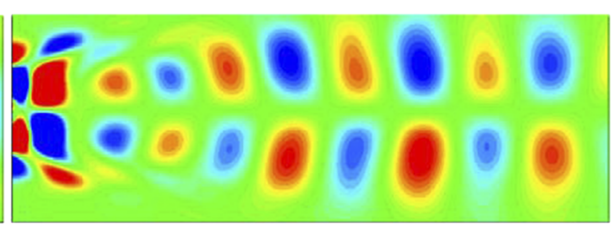

mode8

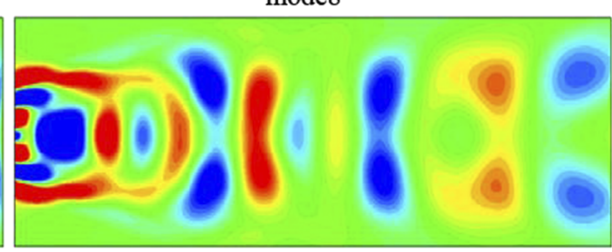

mode10
FIG. 8. Transitional state: (a) spatial structures of vorticity POD modes (modes 1 to 10 from top to bottom), (b) temporal coefficient time histories of vorticity POD modes for the vorticity field, and (c) power spectra of the temporal coefficients of vorticity POD modes. 

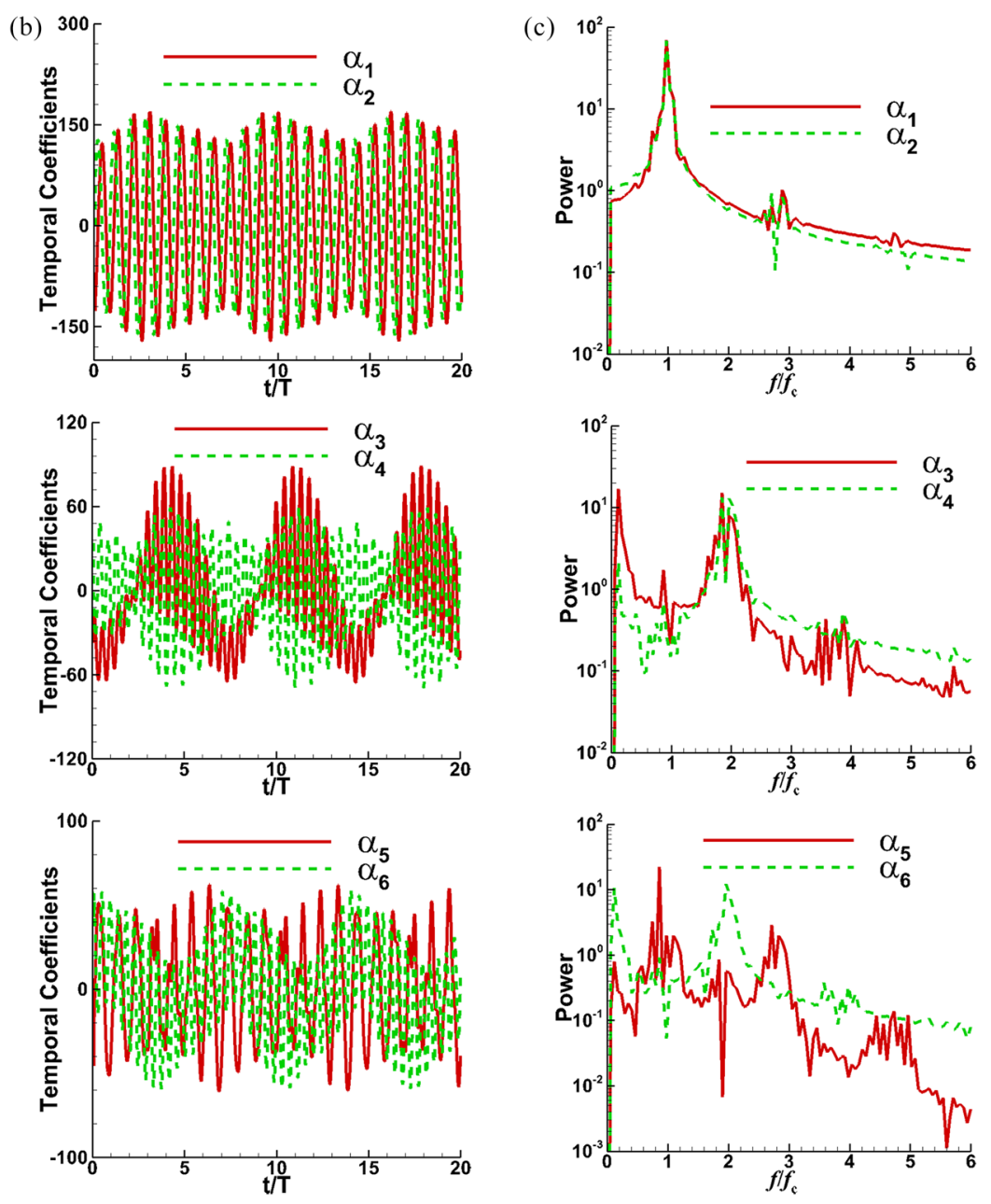

FIG. 8. (Continued).
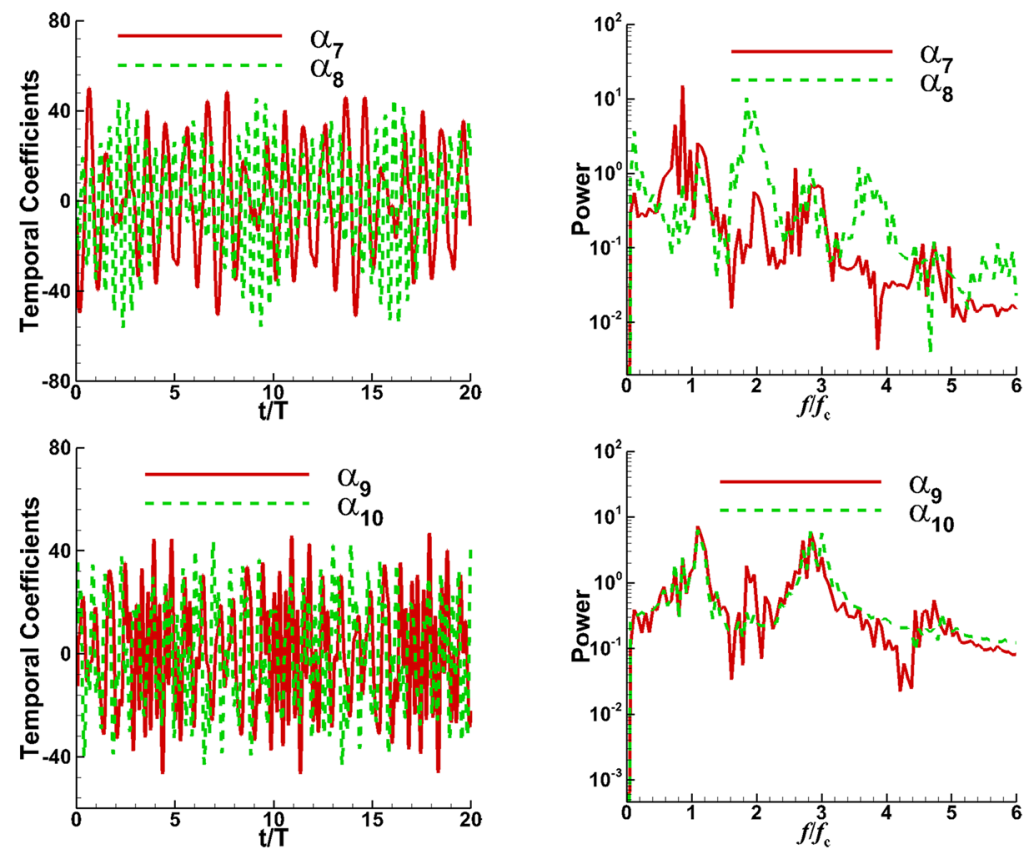

\section{B. The transitional state}

When the system is under the conditions of a critical spacing $S=4$, a higher oscillating amplitude $h=0.35$, and an oscillating frequency close to natural vortex shedding frequency $f_{c}=0.9 f_{n s}$, the transitional state occurs. Figure 6(a) shows the velocity portrait at point $P$. The velocity phase portrait at this point shows some random tendency. The time history of vorticity at point $P$ is shown in Fig. 6(b), and the regular periodicity disappears. Weak chaos is shown in Fig. 6(b). Again, the time-varying part of the flow is selected to be decomposed and the fluctuating fields are represented by 
the coherent structures. The values of the first ten modes with the largest eigenvalues corresponding to the most energetic modes are shown in Table III. The energy of each mode and cumulative energy versus the number of the POD modes are plotted in Fig. 7 for the first 20 modes. Obviously, the first two modes are dominating modes, containing about $35 \%$ and $34 \%$ of the total energy, respectively. The third and fourth modes account for lower energy percentages of less than $5 \%$. The first ten modes collectively contain almost $90 \%$ of the energy. Therefore, with these modes, the majority of flow characteristics can be captured. Figure 7 shows that all the modes are not exactly paired in terms of energy level, not like in the lock-in state in Fig. 3. Thus, if the vortex shedding pattern switches from the lock-in state to the transitional state, the paired modes in the lock-in state cannot be maintained, which has also been discovered by Konstantinidis et al. ${ }^{61}$ for wakes behind a single cylinder.

The first ten POD modes of the coherent structures are shown in Fig. 8(a). It can be seen that the first two modes, modes 1 and 2, are paired in terms of similarity in their spatial structures, although their energy levels are not the same but close as evident in Fig. 7. Both the modes also show a topbottom mirrored symmetry. In addition, the structures of the first two modes are almost the same as the first two modes of the lock-in state, which appear to be traveling-wave-like structures. The third, fourth, sixth, and eighth modes exhibit somewhat antisymmetric properties though not exactly. The fifth, seventh, ninth, and tenth modes show some symmetric properties but again not exactly. However, with flow structure similarity and close energy levels, it seems that modes 4 and 6 , mode 5 and 7, and modes 9 and 10 can still be paired. Modes 3 and 8 are some modes with a different topology and are mainly active during the rapid changes of transition, and this rapid change phenomenon is also found in the wake behind a single cylinder. ${ }^{62,63}$ With these somewhat irregular flow structures, non-periodic motions of the flow start to evolve.

The temporal coefficients of these modes are plotted in Fig. 8(b). The temporal coefficients of the first two modes

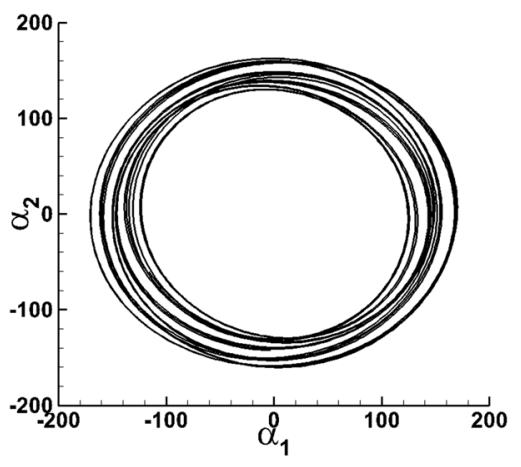

(a)

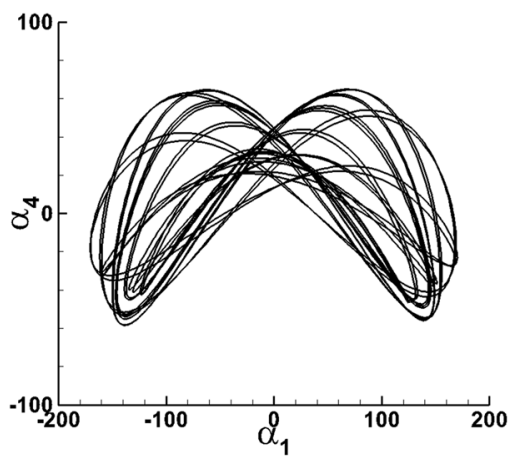

(c)

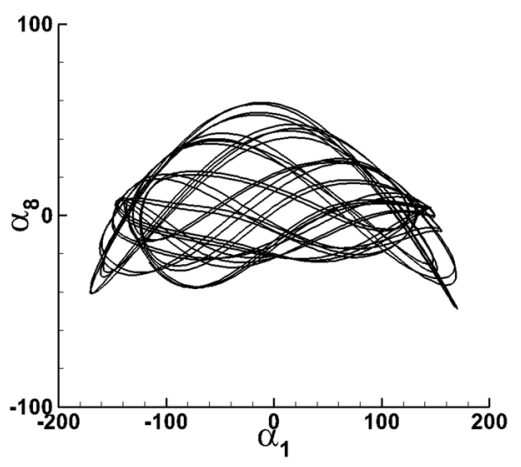

(e)

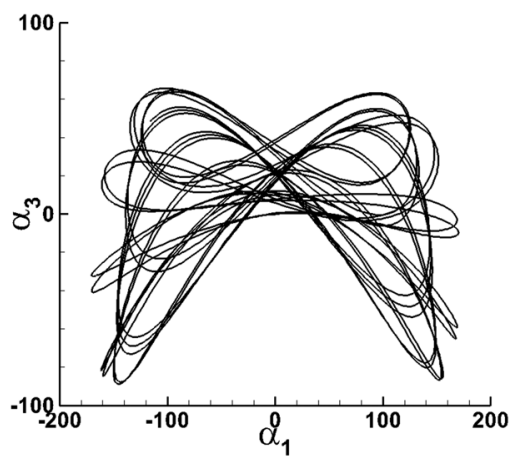

(b)

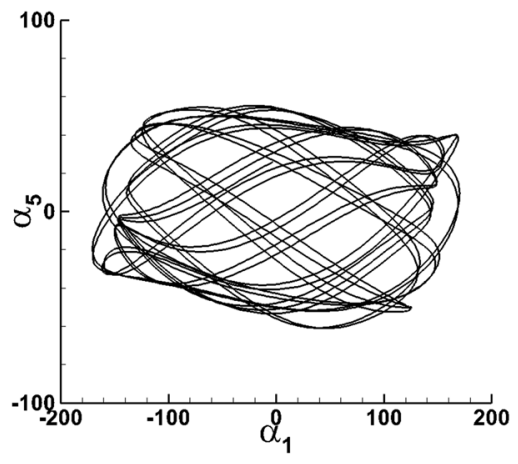

(d)

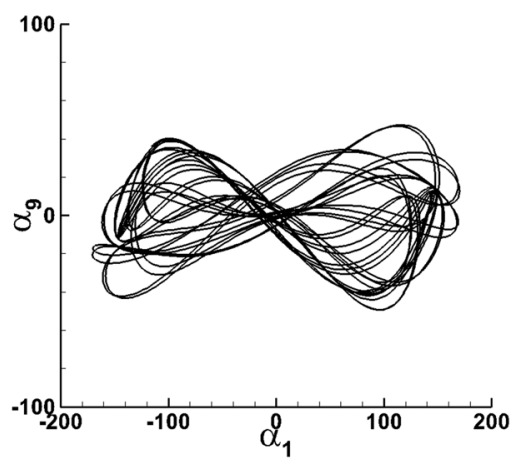

(f)
FIG. 9. Phase portraits of vorticity POD temporal coefficients in the transitional state. (a) $\alpha_{2}$ vs. $\alpha_{1}$, (b) $\alpha_{3}$ vs. $\alpha_{1}$, (c) $\alpha_{4}$ vs. $\alpha_{1}$, (d) $\alpha_{5}$ vs. $\alpha_{1}$, (e) $\alpha_{8}$ vs. $\alpha_{1}$, and (f) $\alpha_{9}$ vs. $\alpha_{1}$. 
TABLE IV. LLE for each vorticity mode in the transitional state.

\begin{tabular}{|c|c|c|c|c|c|c|c|c|c|c|}
\hline Temporal coefficients & $\alpha_{1}$ & $\alpha_{2}$ & $\alpha_{3}$ & $\alpha_{4}$ & $\alpha_{5}$ & $\alpha_{6}$ & $\alpha_{7}$ & $\alpha_{8}$ & $\alpha_{9}$ & $\alpha_{10}$ \\
\hline Lag $\tau$ (time units) & 1.45 & 1.45 & 1.15 & 0.75 & 1.35 & 0.95 & 1.65 & 0.8 & 0.7 & 0.7 \\
\hline Embedding dimension $m$ & 5 & 5 & 5 & 5 & 5 & 5 & 5 & 5 & 5 & 5 \\
\hline LLE (31T-35T) & 0.5625 & 0.5345 & 1.0124 & 0.5123 & 0.4285 & 0.4583 & 0.9584 & 0.9215 & 0.6251 & 0.7521 \\
\hline LLE $(81 T-85 T)$ & 0.0085 & 0.0093 & 0.0334 & 0.0256 & 0.0250 & 0.0451 & 0.0207 & 0.0512 & 0.0287 & 0.0282 \\
\hline $\operatorname{LLE}(81 T-100 T)$ & 0.0084 & 0.0104 & 0.0220 & 0.0254 & 0.0253 & 0.0207 & 0.0205 & 0.0244 & 0.0294 & 0.0295 \\
\hline
\end{tabular}

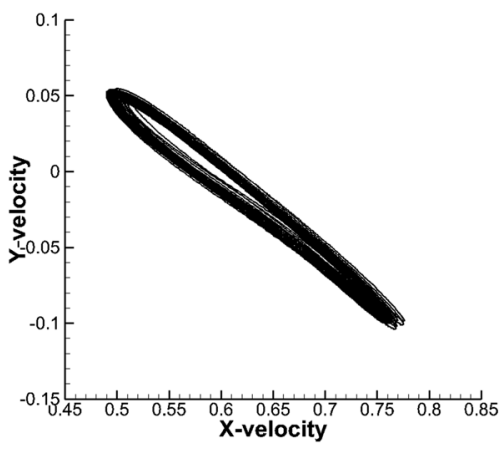

(a)

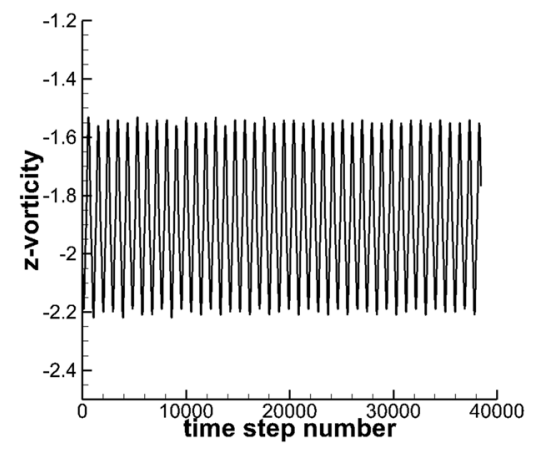

(b)
FIG. 10. Quasiperiodic state: (a) Velocity phase portrait at point $P$ and (b) vorticity time history at point $P$. show clear periodicity while the others do not exhibit regular periodic properties and only quasi-periodicity at most. In the spectral-domain results in Fig. 8(c), the dominant frequency for the first two modes is the same as the forcing frequency, $f_{c}$, corresponding to the downstream-cylinder oscillating frequency. It can be seen that higher modes contain different frequencies. Peaks at $2 f_{c}$ and $2 f_{n s}$ can be found in modes 3 and 4. A peak at $f_{n s}$ can be seen in mode 6 . In addition, peaks with lower amplitudes are found in modes 5, 7, 8, 9, and 10, which may cause some modulations in these frequencies as well. Similar multi-frequencies were discovered in the previous work ${ }^{1,10}$ in the transitional state.

The phase portraits of temporal coefficients of the higher modes versus that of the first mode are displayed in Fig. 9. Unlike the lock-in state, the trajectories of $\alpha_{2}$ versus $\alpha_{1}$ do not show a limit circle but a series of round circles, which means that modes 1 and 2 contain the same dominant frequency. All the others do not show periodicity with a dominant frequency. These results can be observed in Fig. 8(c), where modes with low energy contain different frequencies, which can cause some modulations. ${ }^{10}$

To investigate the behaviors of LLEs in the transitional state, LLEs of the vorticity temporal coefficients for each of the modes are calculated. The development of the LLEs is again evaluated by using the early $5 T$, late $5 T$, and last $20 T$ time coefficient data to calculate the LLEs. ${ }^{60}$ The results are listed in Table IV. In the transitional state, at point
$P, \tau=1.45, m=5$, and LLE $=0.0112$, which means that weak chaos exists in the transitional state. From Table IV, it can be seen that the LLEs become smaller (but remain positive) as time increases from the early $5 T$ to the late $5 T$. The LLEs of the late $5 T$ and last $20 T$ have very close values, except for the modes 3,6 , and 8 . This is because modes 3,6 , and 8 contain low frequencies, which cannot be captured by the late $5 T$ data, but can be captured by the last $20 T$ data. It also indicates that when time increases, the LLEs of each mode become smaller until reaching a constant value, and $20 T$ is long enough to capture the features in the transitional state in this study.

The different LLEs indicate different chaos levels of these modes. After a long period of time, the LLEs for the modes with lower energy become almost the same. Table IV shows that after a long period of time, the LLEs of the first two modes are close to zero (around 0.0112), which means that the chaos levels of point $P$ and the first two energetic modes are in the same order and the first two modes are relatively stable. The LLEs of the other modes are all positive and larger than 0.0112 , which shows that their chaos levels are higher than the first two modes and weak chaotic motions exist in these modes. It again demonstrates that $20 T$ is long enough to capture the features in the transitional state because the LLEs reach a constant value. The embedding dimensions of all the modes are the same as that of the vorticity history at point $P$, while only the lags for the first two dominant modes are the same as those of point

TABLE V. Energy levels of the POD modes and their respective contributions to the total energy in the quasiperiodic state.

\begin{tabular}{lccccccrr}
\hline \hline Modes & 1 & 2 & 3 & 4 & 5 & 6 & \multicolumn{1}{c}{7} & \multicolumn{1}{c}{8} \\
\hline Energy of each mode (\%) & 24.45 & 22.51 & 20.43 & 17.84 & 3.33 & 2.97 & 1.58 & 1.50 \\
Cumulative energy (\%) & 24.45 & 46.96 & 67.39 & 85.23 & 88.56 & 91.53 & 93.11 & 94.61 \\
\hline \hline
\end{tabular}




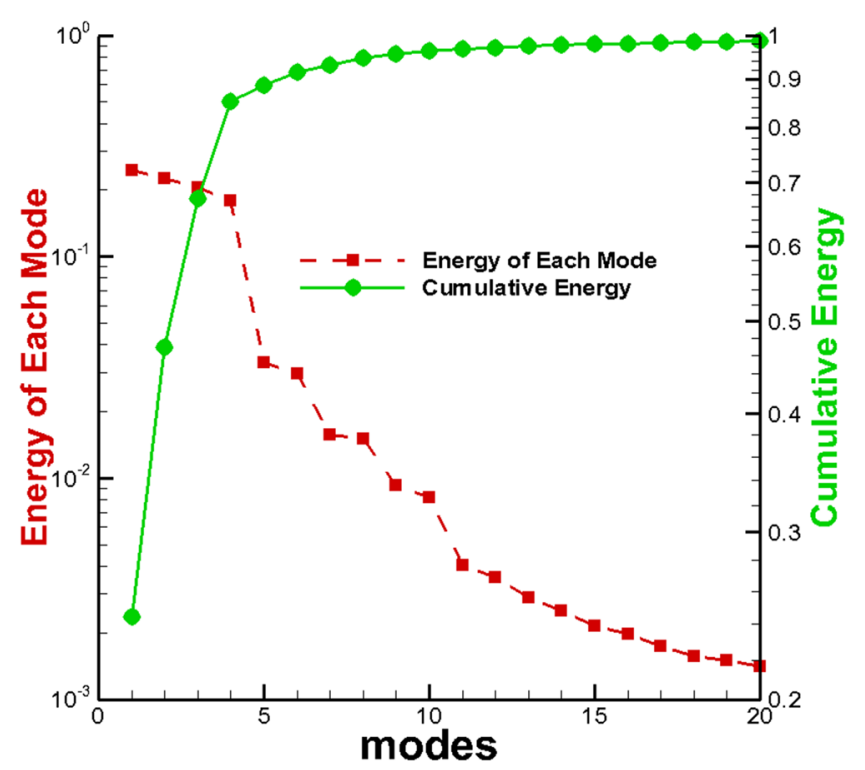

FIG. 11. Energy distributions of the first 20 POD modes in the quasiperiodic state.

$P$. Thus, it can be conjectured that in the transitional state, the first two dominant coherent structures are relatively stable, while weak chaos exists in the higher modes and leads the higher-mode structures to become unstable first. After a long period of time, the LLEs of the first two modes are almost the same with that of the vorticity history at point $P$. It is without coincidence that the lag time listed in Table IV is again the same for the paired modes, modes 1 and 2 and modes 9 and 10 , with a trend to decrease in the higher modes. On the other hand, the fact that different POD modes have different LLEs may provide an evidence that there is no single LLE for the entire flow field, ${ }^{64}$ but there can still be an LLE for each of the POD modes.

To check the chaos level of the transitional state, we also tested another transitional state in our previous work: ${ }^{1}$ $S=4, h=0.15$, and $f_{c}=1.1 f_{n s}$. The time histories of z-vorticity at point $P$ also show non-periodicity. For a long period of time, the LLEs are also positive in the modes with low energy, providing another evidence that chaos exists in the transitional state, especially in the low energy modes.

\section{The quasiperiodic state}

When increasing the oscillating frequency in the lock-in state to $f_{c}=1.7 f_{n s}$, still at the vortex suppression spacing $S=2$, but with a smaller oscillating amplitude $h=0.15$, the quasiperiodic state occurs. Figure 10(a) shows the velocity portrait at point $P$, which displays a quasiperiodic pattern. The time history of vorticity at point $P$ is shown in Fig. 10(b) where an obviously periodic property can be seen. Again, the first eight modes corresponding to the first eight largest eigenvalues are the most energetic modes, as listed in Table V. The plots of the energy of each mode and cumulative energy versus the number of the POD modes are shown in Fig. 11 for the first 20 modes. Apparently, the first four modes are dominant modes, which contain more than $85 \%$ of the total energy. The fifth and sixth modes account for a lower energy percentage of less than $4 \%$. The first eight modes collectively contain more than $95 \%$ of the energy. Although Fig. 11 does not clearly indicate paired modes as those found in the lock-in state, similarity in the mode spatial structures between the neighboring (a)

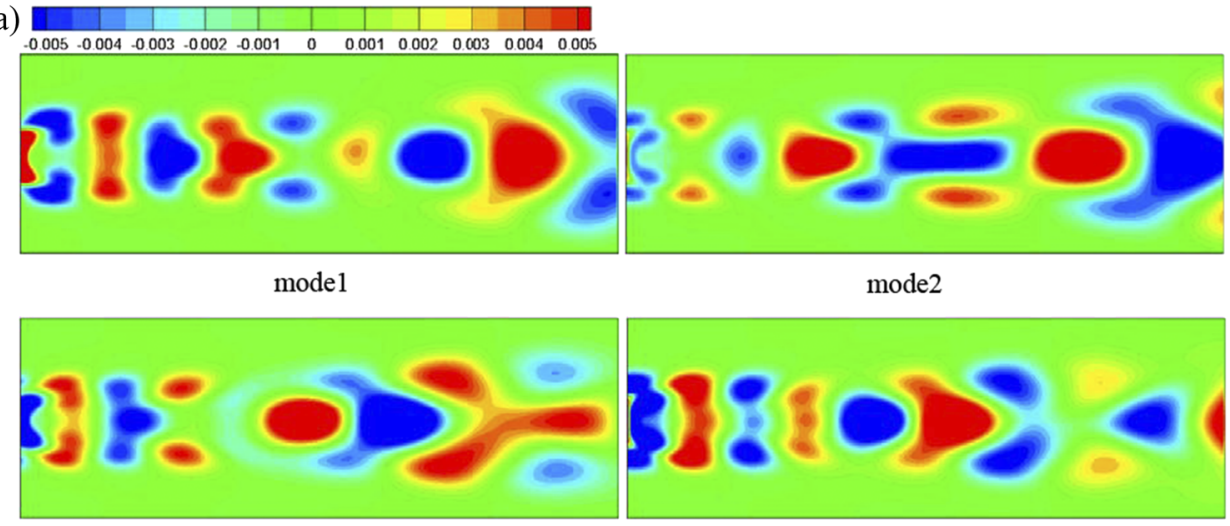

mode3

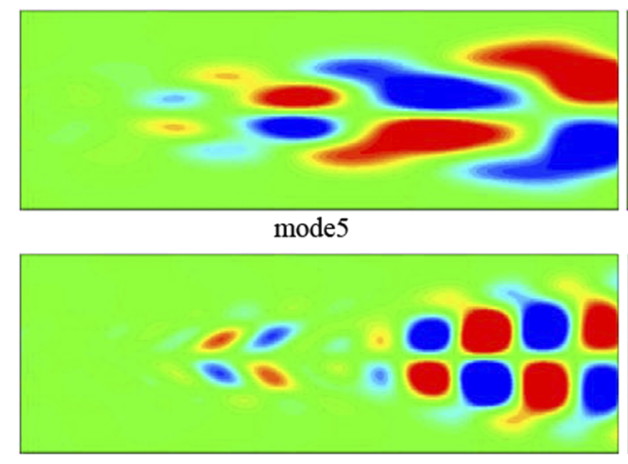

mode 7 mode4

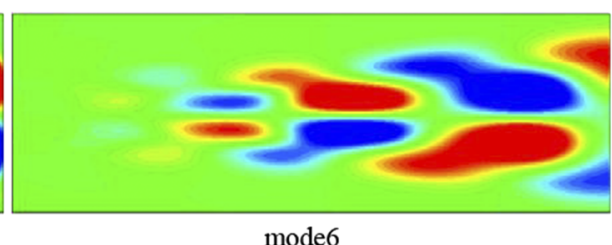

mode6

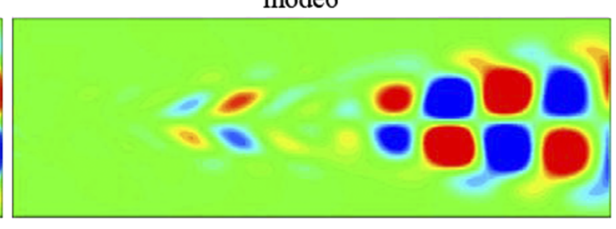

mode8
FIG. 12. Quasiperiodic state: (a) spatial structures of vorticity POD modes (modes 1 to 8 from top to bottom), (b) temporal coefficient time histories of vorticity POD modes for the vorticity field, and (c) power spectra of the temporal coefficients of vorticity POD modes. 
(b)
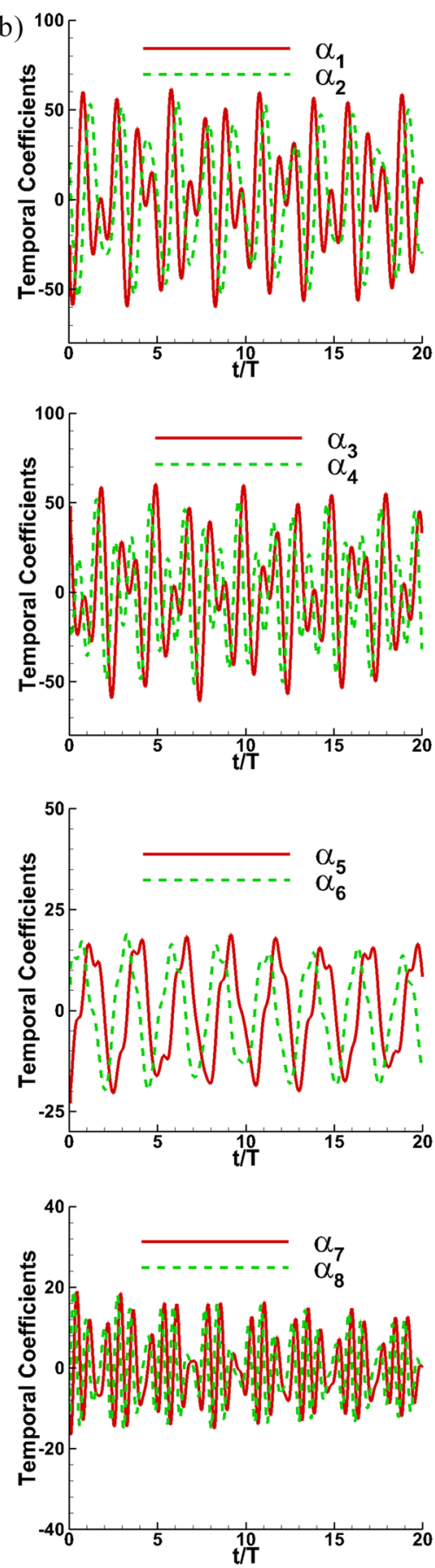
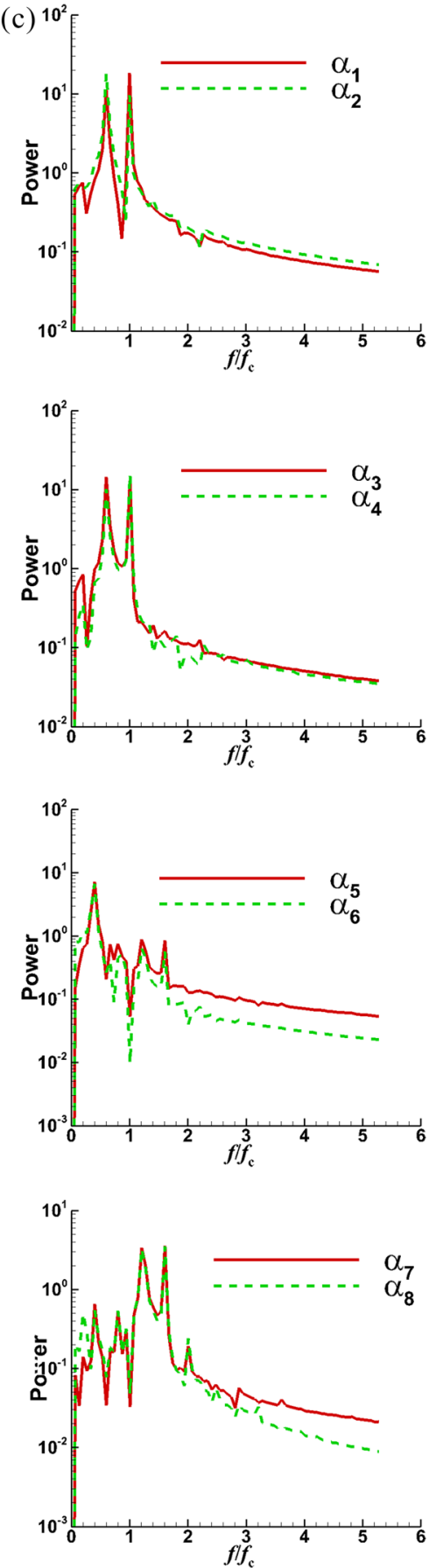

modes, shown in Fig. 12(a), brings back the odd-even pair patterns as in the lock-in state. However, the coherent structures in the quasiperiodic state are very different from those in the lock-in state. Anyway, it seems that the pattern of paired modes re-emerges when the flow reaches the quasiperiodic state.

The first eight POD modes are shown in Fig. 12(a). It can be seen that the first two modes show the top-bottom mirrored symmetry. The third and fourth modes also exhibit the top-bottom symmetry, although variations in the modestructure contours make this symmetry inexact. The next four modes with less energy show antisymmetry, again not in an exact way. An interesting phenomenon is that for the last four modes, there is weaker vorticity in the near wake than in the far wake. The temporal coefficients of these modes are plotted in the middle column of Fig. 12(b). Clearly, the temporal coefficients of all the dominant modes in this state are non-periodic, even for the most energetic modes. The dominant frequencies for modes $1,2,3$, and 4 are $f_{c}$ and $f_{n s}$, respectively. Thus, the first four modes contain the fundamental frequency corresponding to the oscillating frequency and the vortex shedding frequency. The dominant frequency for modes 5 and 6 is $f_{c}-f_{n s}$, corresponding to the linear combination of vortex shedding frequency and oscillating frequency. For the temporal coefficients of the last two modes, modes 7 and 8 , peaks are identified at $2 f_{n s}, f_{c}-f_{n s}$, and $2 f_{c}$. Therefore, the quasiperiodic state exhibits clearly multiple peaks at two frequencies $f_{c}$ and $f_{n s}$ and their linear combinations, which is similar to 


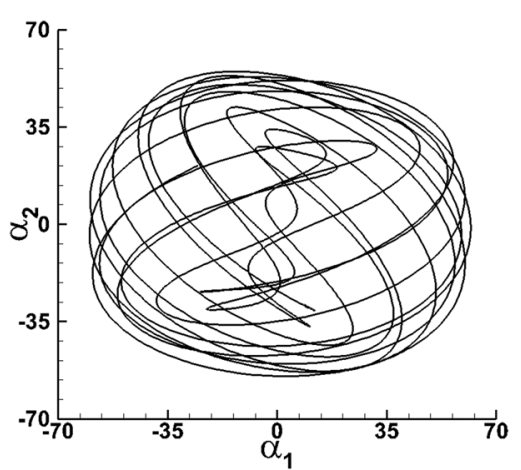

(a)

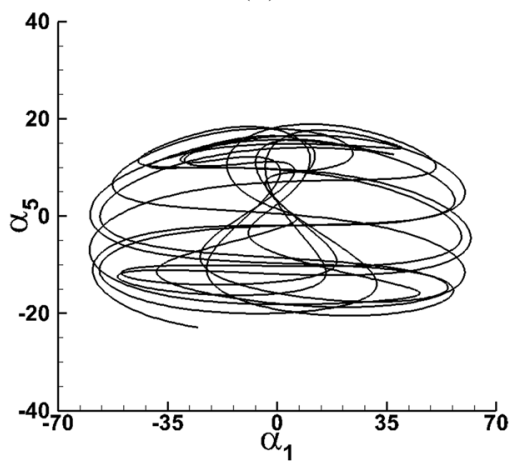

(c)

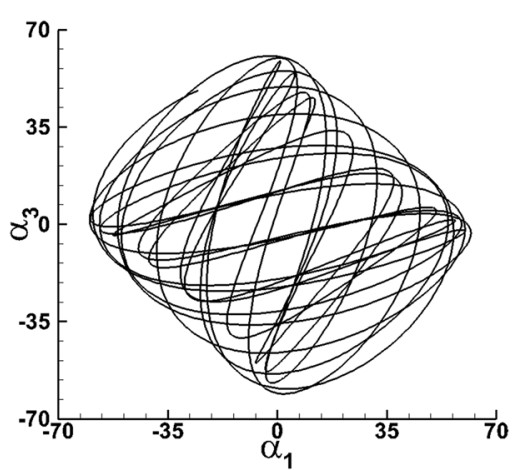

(b)

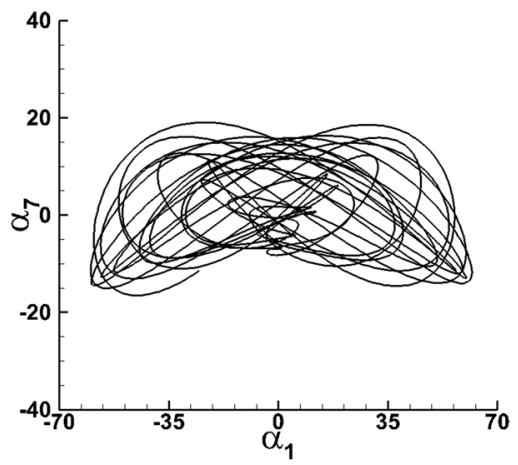

(d)
FIG. 13. Phase portraits of vorticity POD temporal coefficients in the quasiperiodic state. (a) $\alpha_{2}$ vs. $\alpha_{1}$, (b) $\alpha_{3}$ vs. $\alpha_{1}$, (c) $\alpha_{5}$ vs. $\alpha_{1}$, and (d) $\alpha_{7}$ vs. $\alpha_{1}$. the property of the double-frequency quasiperiodic system discussed in the previous study. ${ }^{64}$

The phase portraits of temporal coefficients of the higher modes versus that of the first mode are shown in Fig. 13. No clear closed circles can be seen as those in the lock-in state shown in Fig. 5(a). The multi-frequency periodicity, as evident in Fig. 12(c) where each mode contains multiple dominant frequencies, cannot be seen in such phase portraits using $\alpha_{1}$ as the reference. This is because $\alpha_{1}$ itself contains two frequencies in addition to phase shifts from all the other mode coefficients. Therefore, even with double-frequency periodicity in the quasiperiodic state, the phase portraits in Fig. 13 do not show clear attractors of the system.

The LLEs of the vorticity POD temporal coefficients are calculated to explore the time evolution of the POD modes. The LLEs of the early $5 T$, late $5 T$, and last $20 T$ time coefficients are calculated with the results shown in Table VI. The LLE of the vorticity history at point $P$ is also calculated to compare with the LLE of each mode, with the results being $\tau=1.5, m=5$, and LLE $=-0.0024$, which means that the quasiperiodic state is stable. Table VI shows that the LLEs in the quasiperiodic state change from positive to negative (close to zero) as time increases from the early $5 T$ to the late $5 T$. Change of LLEs in time from positive to negative in a quasiperiodic state was also found by Glaz et al. ${ }^{60}$ The LLEs of the late $5 T$ and the last $20 T$ are very close, except for mode 8. This is because mode 8 contains low frequencies, which cannot be captured by the late $5 T$ data but by the last $20 T$ data. It also indicates that when time increases, the LLE of each mode becomes smaller until reaching a constant value, and $20 T$ is long enough to capture the time-development feature of the POD time coefficients in the quasiperiodic state. From Table VI, it can be seen that, for a long period of time, the LLEs of all the modes are close to -0.0024 , the same as the LLE of vorticity history at point $P$. The embedding dimensions of all the modes are also the same as that of point $P$, while only the lag of the first four modes is the same as that of point $P$. As the LLEs of all the modes are negative, stable motions exist in all the POD coherent structures in the quasiperiodic state, which is also shown in the previous study. ${ }^{64}$ The lag time in each of the mode pairs is the same, as observed in the lock-in state, but does not decrease monotonically with higher modes.

To recheck the above revealed chaotic behaviors of the periodic state, we tested another quasiperiodic state according

TABLE VI. LLE for each vorticity mode in the quasiperiodic state.

\begin{tabular}{|c|c|c|c|c|c|c|c|c|}
\hline Temporal coefficients & $\alpha_{1}$ & $\alpha_{2}$ & $\alpha_{3}$ & $\alpha_{4}$ & $\alpha_{5}$ & $\alpha_{6}$ & $\alpha_{7}$ & $\alpha_{8}$ \\
\hline Lag $\tau$ (time units) & 1.5 & 1.5 & 1.5 & 1.5 & 2 & 2 & 0.75 & 0.75 \\
\hline Embedding dimension $m$ & 5 & 5 & 5 & 5 & 5 & 5 & 5 & 5 \\
\hline $\operatorname{LLE}(31 T-35 T)$ & 0.2814 & 0.2587 & 0.2726 & 0.2825 & 0.5588 & 0.4435 & 0.5657 & 0.5489 \\
\hline LLE $(81 T-85 T)$ & -0.0023 & -0.0023 & -0.0024 & -0.0024 & -0.0024 & -0.0024 & -0.0025 & -0.0041 \\
\hline LLE $(81 T-100 T)$ & -0.0024 & -0.0024 & -0.0024 & -0.0024 & -0.0025 & -0.0025 & -0.0025 & -0.0025 \\
\hline
\end{tabular}


to the previous study: ${ }^{1} S=6, h=0.15$, and $f_{c}=1.3 f_{n s}$. The time history of vorticity at point $P$ also shows quasiperiodicity. For a long period of time, the LLEs also tend to zero and the LLEs values for each mode are close to the LLE of vorticity history at point $P$. This confirms that the long-time dynamic behavior of the quasiperiodic state is stable. ${ }^{10}$

\section{CONCLUSIONS}

For a multi-variable dynamic system of flow around two tandem cylinders with the downstream cylinder oscillating transversely, there exist three states of nonlinear response: lock-in, transitional, and quasiperiodic. In this study, these three states are further explored using the POD analysis and the LLEs of the POD mode coefficients along with the LLE of the vorticity history at a specific point in the flow field. The POD analysis expands the unsteady flow solution into a series summation of each spatial-only POD mode with a temporal coefficient. The spatial coherent flow structures are represented by the POD modes, and the time evolutions of these structures are represented by their corresponding temporal coefficients.

We focus on the first eight or ten POD modes because they contain almost $90 \%$ total energy of flow in these three states. The paired POD modes, i.e., the two adjacent modes containing almost the same energy with a similar POD spatial pattern, can be found in all the three states, although the typical odd-even pair modes in the transitional state are not quite clearly identified. The flow structures of the POD modes are sometimes symmetric or anti-symmetric with respect to the horizontal centerline of the tandem cylinder system. The first pair (modes 1 and 2) is symmetric in all three states and contains almost $70 \%$ of energy in the lock-in and transitional states but only around $50 \%$ in the quasiperiodic state. The second pair (modes 3 and 4) is antisymmetric in the lock-in state and still symmetric, although not exactly, in the quasiperiodic state. In the transitional state, modes 3 and 4 are not paired, with mode 3 showing neither symmetry nor antisymmetry. Instead, paired modes are found between modes 4 and 6 , and also modes 9 and 10. The first four modes (first two pairs) contain more than $92 \%$ of energy in the lock-in state and more than $80 \%$ of energy in the quasiperiodic state. After the first two pairs, the odd pairs exhibit symmetry and even pairs exhibit antisymmetry in the lock-in state, and all the modes show the antisymmetry (although not exactly) in the quasiperiodic state. The transitional state possesses neither exactly symmetry nor antisymmetry in all the higher modes other than the first pair.

The temporal coefficients for the lock-in POD modes show regular periodicity with dominant frequencies at $f_{c}, 2 f_{c}$, $3 f_{c}$, and $4 f_{c}$, for the first to fourth pairs of modes, respectively. The phase portraits for the vorticity POD temporal coefficients of the higher modes versus that of the first mode are all single closed-loop curves. The phase portraits of the temporal coefficients of the velocity POD modes are all clean, closed ellipses. The temporal coefficients of the first two modes in the transitional state exhibit clear periodicity, while the others do not exhibit regular periodic properties. Prominent peaks in the transitional state are at $f_{c}, 2 f_{c}$, and $2 f_{n s}$. The trajectories of $\alpha_{2}$ versus $\alpha_{1}$ show a series of round circles. The phase portraits of temporal coefficients of the others are not clean and show some chaotic behaviors. The temporal coefficients of all the dominant modes in the quasiperiodic state show doublefrequency periodicity. As $\alpha_{1}$ itself contains two frequencies with phase shifts, the phase portraits do not show clear attractors of the system. The spectral peaks are at $f_{c}, f_{n s}$ and the linear combinations of $f_{c}$ and $f_{n s}$.

By looking into the LLEs of the POD coefficients and the vorticity history at point $P$, we find that the LLEs in the lock-in state for all the POD modes and vorticity at point $P$ are zero, which means that there is no unstable development in the lockin state. In the transitional state, the LLEs evolve with time from larger values to smaller values, but they are still larger than zero after a long period of time. This indicates that when flow becomes more periodic, the LLE of each mode becomes smaller. The LLEs for the first two modes are almost the same with that of point $P$ and are close to zero, which means that almost no chaos exists in those two modes of large coherent structures in the transitional state. In this state, weak chaos exists in other structures with almost the same chaos level as indicated by the values of their LLEs. In the quasiperiodic state, the LLEs developed from positive to negative after a long period of time. In addition, the LLEs of all the POD modes and that of point $P$ are almost the same with a value no larger than zero (but close to zero), which means that the motions in the quasiperiodic state are close to those in the lock-in state and the system is stable for the quasiperiodic state. It is also that noted for all three states, the values of lag time of the two modes in a pair are the same or very close, and the lag time of the vorticity history at point $P$ is almost the same as the lag time of the first two dominant modes. The embedding dimensions of all the modes are the same as that of the vorticity history at point $P$ in all the nonlinear states.

It should be noted that in this study, we mostly truncate the POD modes up to the first 10 modes. The behavior of higherorder modes can possibly be different from those of the first 10. Although the dynamics of higher-order POD modes may also influence the dynamics of flow, this study mostly focuses on the dynamics of lower-order modes whose higher-energy levels are likely to be responsible for the significant dynamic development of flow.

Finally, although this study is based on flow simulation at a Reynolds number of 100, the above conclusions are applicable for flow in the laminar regime in the same non-linear states for similar types of systems.

${ }^{1}$ X. Yang and Z. C. Zheng, "Nonlinear spacing and frequency effects of an oscillating cylinder in the wake of a stationary cylinder," Phys. Fluids 22, 043601 (2010).

${ }^{2}$ D. Sumner, "Two circular cylinders in cross-flow: A review," J. Fluids Struct. 26, 849 (2010).

${ }^{3}$ A. Chatterjee, "An introduction to the proper orthogonal decomposition," Curr. Sci. 78, 808 (2000).

${ }^{4}$ K. Taira, S. L. Brunton, S. T. Dawson, C. W. Rowley, T. Colonius, B. J. McKeon, O. T. Schmidt, S. Gordeyev, V. Theofilis, and L. S. Ukeiley, "Modal analysis of fluid flows: An overview," AIAA J. 55, 4013 (2017).

${ }^{5}$ M. M. Zdravkovich, "The effects of interference between circular cylinders in cross flow," J. Fluids Struct. 1, 239 (1987).

${ }^{6}$ B. Qin, M. M. Alam, and Y. Zhou, "Two tandem cylinders of different diameters in cross-flow: Flow-induced vibration," J. Fluid Mech. 829, 621 (2017). 
${ }^{7}$ L. Wang, M. M. Alam, and Y. Zhou, "Two tandem cylinders of different diameters in cross-flow: Effect of an upstream cylinder on wake dynamics," J. Fluid Mech. 836, 5 (2018).

${ }^{8}$ J. Li, A. Chambarel, M. Donneaud, and R. Martin, "Numerical study of laminar flow past one and two circular cylinders," Comput. Fluids 19, 155 (1991).

${ }^{9}$ R. J. Jiang and J. Z. Lin, "Poiseuille flow-induced vibrations of two tandem circular cylinders with different mass ratios," Phys. Fluids 28, 064105 (2016).

${ }^{10}$ G. V. Papaioannou, D. K. Yue, M. S. Triantafyllou, and G. E. Karniadakis, "Evidence of holes in the Arnold tongues of flow past two oscillating cylinders," Phys. Rev. Lett. 96, 014501 (2006).

${ }^{11}$ E. Wang, Q. Xiao, Q. Zhu, and A. Incecik, "The effect of spacing on the vortex-induced vibrations of two tandem flexible cylinders," Phys. Fluids 29, 077103 (2017).

${ }^{12}$ L. Lin, X. Guo, G. Tang, M. Liu, C. Chen, and Z. Xie, "Numerical investigation of flow-induced rotary oscillation of circular cylinder with rigid splitter plate," Phys. Fluids 28, 093604 (2016).

${ }^{13}$ B. S. Carmo, J. R. Meneghini, and S. J. Sherwin, "Secondary instabilities in the flow around two circular cylinders in tandem," J. Fluid Mech. 644, 395 (2010).

${ }^{14}$ I. AlQadi, M. AlHazmy, A. Al-Bahi, and W. Rodi, "Large eddy simulation of flow past tandem cylinders in a channel," Flow, Turbul. Combust. 95, 621 (2015).

${ }^{15}$ M. D. Griffith, D. L. Jacono, J. Sheridan, and J. S. Leontini, "Flow-induced vibration of two cylinders in tandem and staggered arrangements," J. Fluid Mech. 833, 98 (2017).

${ }^{16}$ Y. Tanida, A. Okajima, and Y. Watanabe, "Stability of a circular cylinder oscillating in uniform flow or in a wake," J. Fluid Mech. 61, 769 (1973).

${ }^{17} \mathrm{G}$. Xu and Y. Zhou, "Strouhal numbers in the wake of two inline cylinders," Exp. Fluids 37, 248 (2004)

${ }^{18} \mathrm{~J} . \mathrm{Li}, \mathrm{J}$. Sun, and B. Roux, "Numerical study of an oscillating cylinder in uniform flow and in the wake of an upstream cylinder," J. Fluid Mech. 237, 457 (1992)

${ }^{19}$ N. Mahir and D. Rockwell, "Vortex formation from a forced system of two cylinders. Part I: Tandem arrangement," J. Fluids Struct. 10, 473 (1996).

${ }^{20}$ G. V. Papaioannou, D. K. Yue, M. S. Triantafyllou, and G. E. Karniadakis, "Three-dimensionality effects in flow around two tandem cylinders," J. Fluid Mech. 558, 387 (2006).

${ }^{21}$ S. Bao, S. Chen, Z. Liu, J. Li, H. Wang, and C. Zheng, "Simulation of the flow around an upstream transversely oscillating cylinder and a stationary cylinder in tandem," Phys. Fluids 24, 023603 (2012).

${ }^{22} \mathrm{Z}$. X. Liang and H. B. Dong, "On the symmetry of proper orthogonal decomposition modes of a low-aspect-ratio plate," Phys. Fluids 27, 063601 (2015).

${ }^{23}$ G. Riches, R. Martinuzzi, and C. Morton, "Proper orthogonal decomposition analysis of a circular cylinder undergoing vortex-induced vibrations," Phys. Fluids 30, 105103 (2018).

${ }^{24} \mathrm{~F}$. Takens, "Detecting strange attractors in turbulence," in Dynamical Systems and Turbulence, Warwick 1980 (Springer, 1981), p. 366.

${ }^{25}$ M. T. Rosenstein, J. J. Collins, and C. J. De Luca, "A practical method for calculating largest Lyapunov exponents from small data sets," Physica D 65, 117 (1993)

${ }^{26}$ Z. A. Wei, Z. C. Zheng, and X. Yang, "Computation of flow through a threedimensional periodic array of porous structures by a parallel immersedboundary method," J. Fluids Eng. 136, 040905 (2014).

${ }^{27}$ N. Aubry, P. Holmes, J. L. Lumley, and E. Stone, "The dynamics of coherent structures in the wall region of a turbulent boundary layer," J. Fluid Mech. 192, 115 (1988).

${ }^{28}$ I. Mezic, "Analysis of fluid flows via spectral properties of the Koopman operator," Annu. Rev. Fluid Mech. 45, 357 (2013).

${ }^{29}$ P. Holmes, Turbulence, Coherent Structures, Dynamical Systems and Symmetry (Cambridge University Press, 1998).

${ }^{30}$ A. E. Deane, I. G. Kevrekidis, G. E. Karniadakis, and S. A. Orszag, "Lowdimensional models for complex geometry flows: Application to grooved channels and circular cylinders," Phys. Fluids A 3, 2337 (1991).

${ }^{31}$ G. Berkooz, P. Holmes, and J. L. Lumley, "The proper orthogonal decomposition in the analysis of turbulent flows," Annu. Rev. Fluid Mech. 25, 539 (1993).

${ }^{32}$ J. Malm, P. Schlatter, and D. S. Henningson, "Coherent structures and dominant frequencies in a turbulent three-dimensional diffuser," J. Fluid Mech. 699, 320 (2012).
${ }^{33}$ S. Roy, J. C. Hua, W. Barnhill, G. H. Gunaratne, and J. R. Gord, "Deconvolution of reacting-flow dynamics using proper orthogonal and dynamic mode decompositions," Phys. Rev. E 91, 013001 (2015).

${ }^{34}$ A. Wolf, J. B. Swift, H. L. Swinney, and J. A. Vastano, "Determining Lyapunov exponents from a time series," Physica D 16, 285 (1985).

${ }^{35}$ F. Ginelli, P. Poggi, A. Turchi, H. Chate, R. Livi, and A. Politi, "Characterizing dynamics with covariant Lyapunov vectors," Phys. Rev. Lett. 99, 130601 (2007).

${ }^{36}$ G. Huang, W. Lu, J. Zhu, X. Fu, and J. Wang, "A nonlinear dynamic model for unsteady separated flow control and its mechanism analysis," J. Fluid Mech. 826, 942 (2017).

${ }^{37}$ A. Guha and F. E. Udwadia, "Nonlinear dynamics induced by linear wave interactions in multilayered flows," J. Fluid Mech. 816, 412 (2017).

${ }^{38}$ A. Towne, O. T. Schmidt, and T. Colonius, "Spectral proper orthogonal decomposition and its relationship to dynamic mode decomposition and resolvent analysis," J. Fluid Mech. 847, 821 (2018).

${ }^{39} \mathrm{~N}$. Zhang and Z. C. Zheng, "An improved direct-forcing immersedboundary method for finite difference applications," J. Comput. Phys. 221, 250 (2007).

${ }^{40}$ Y. Xu, Z. C. Zheng, and D. K. Wilson, "Simulation of turbulent wind noise reduction by porous windscreens using high-order schemes," J. Comput. Acoust. 18, 321 (2010)

${ }^{41}$ X. B. Chen, P. Yu, S. H. Winoto, and H. T. Low, "Numerical analysis for the flow past a porous square cylinder based on the stress-jump interfacial-conditions," Int. J. Numer. Methods Heat Fluid Flow 18, 635 (2008).

${ }^{42}$ Z. A. Wei and Z. C. Zheng, "Fluid-structure interaction simulation on energy harvesting from vortical flows by a passive heaving foil," J. Fluids Eng. 140, 011105 (2018)

${ }^{43}$ R. Borges, M. Carmona, B. Costa, and W. S. Don, "An improved weighted essentially non-oscillatory scheme for hyperbolic conservation laws," J. Comput. Phys. 227, 3191 (2008).

${ }^{44}$ J. C. Adams, "Mudpack: Multigrid portable Fortran software for the efficient solution of linear elliptic partial differential equations," Appl. Math. Comput. 34, 113 (1989).

${ }^{45}$ Z. C. Zheng and Z. A. Wei, "Study of mechanisms and factors that influence the formation of vortical wake of a heaving airfoil," Phys. Fluids 24, 103601 (2012)

${ }^{46} \mathrm{~L}$. Sirovich, "Turbulence and the dynamics of coherent structures. I. Coherent structures," Q. Appl. Math. 45, 561 (1987).

${ }^{47} \mathrm{R}$. Grappin and J. Leorat, "Lyapunov exponents and the dimension of periodic incompressible Navier-Stokes flows: Numerical measurements," J. Fluid Mech. 222, 61 (1991)

${ }^{48} \mathrm{~K}$. Kanno and A. Uchida, "Finite-time Lyapunov exponents in time-delayed nonlinear dynamical systems," Phys. Rev. E 89, 032918 (2014).

${ }^{49}$ H. Kantz and T. Schreiber, Nonlinear Time Series Analysis (Cambridge University Press, 2004), Vol. 7.

${ }^{50}$ N. H. Packard, J. P. Crutchfield, J. D. Farmer, and R. S. Shaw, "Geometry from a time series," Phys. Rev. Lett. 45, 712 (1980).

${ }^{51}$ A. M. Fraser and H. L. Swinney, "Independent coordinates for strange attractors from mutual information," Phys. Rev. A 33, 1134 (1986).

${ }^{52}$ S. Kodba, M. Perc, and M. Marhl, "Detecting chaos from a time series," Eur. J. Phys. 26, 205 (2004).

${ }^{53}$ S. S. Tamil and S. R. Samuel, "Application of Lyapunov exponent on the predictability of temperature over an equatorial station-Chennai," Bull. Environ., Pharmacol. Life Sci. 4, 144 (2015).

${ }^{54}$ M. Perc, "The dynamics of human gait," Eur. J. Phys. 26, 525 (2005).

${ }^{55}$ A. M. Kowalski, M. T. Martin, A. Plastino, and G. Judge, "On extracting probability distribution information from time series," Entropy 14, 1829 (2012)

${ }^{56}$ H. A. Sturges, "The choice of a class interval," J. Am. Stat. Assoc. 21, 65 (1926).

${ }^{57}$ L. Y. Cao, "Practical method for determining the minimum embedding dimension of a scalar time series," Physica D 110, 43 (1997).

${ }^{58}$ D. Rempfer and H. F. Fasel, "Evolution of three-dimensional coherent structures in a flat-plate boundary layer," J. Fluid Mech. 260, 351 (1994).

${ }^{59}$ N. Nabatian, X. Xu, and N. Mureithi, "POD analysis of three-dimensional harmonically forced wake flow of a circular cylinder," Trans. Can. Soc. Mech. Eng. 39, 789 (2015).

${ }^{60}$ B. Glaz, I. Mezić, M. Fonoberova, and S. Loire, "Quasi-periodic intermittency in oscillating cylinder flow," J. Fluid Mech. 828, 680 (2017) 
${ }^{61}$ E. Konstantinidis, S. Balabani, and M. Yianneskis, "Bimodal vortex shedding in a perturbed cylinder wake," Phys. Fluids 19, 011701 (2007).

${ }^{62}$ B. R. Noack, W. Stankiewicz, M. Morzyński, and P. J. Schmid, "Recursive dynamic mode decomposition of transient and post-transient wake flows," J. Fluid Mech. 809, 843 (2016).
${ }^{63}$ B. R. Noack, K. Afanasiev, M. Morzyński, G. Tadmor, and F. Thiele, "A hierarchy of low-dimensional models for the transient and post-transient cylinder wake," J. Fluid Mech. 497, 335 (2003).

${ }^{64}$ H. G. Schuster and W. Just, Deterministic Chaos: An Introduction (John Wiley \& Sons, 2006). 